\title{
One-year pacing dependency after pacemaker implantation in patients undergoing transcatheter aortic valve implantation: Systematic review and meta-analysis
}

Justine M. Ravaux, MD, ${ }^{a}$ Michele Di Mauro, MD, PhD, MSc Biostat, ${ }^{\mathrm{a}}$ Kevin Vernooy, MD, PhD, ${ }^{\mathrm{b}, \mathrm{c}, \mathrm{d}}$ Arnoud W. Van't Hof, MD, PhD, ${ }^{\text {b,c }}$ Leo Veenstra, MD, ${ }^{b}$ Suzanne Kats, MD, PhD, ${ }^{a}$ Jos G. Maessen, $\mathrm{MD}, \mathrm{PhD},{ }^{\mathrm{a}, \mathrm{c}}$ and Roberto Lorusso, $\mathrm{MD}, \mathrm{PhD}^{\mathrm{a}, \mathrm{c}}$

\section{ABSTRACT}

Objectives: Atrioventricular conductions disturbances, requiring permanent pacemaker implantation (PPI), represent a potential complication after transcatheter aortic valve implantation (TAVI), However, little is known about the pacemaker dependency after PPI in this patient setting. This systematic review analyses the incidence of PPI, the short-term (1-year) pacing dependency, and predictors for such a state after TAVI.

Methods: We performed a systematic search in PUBMED, EMBASE, and MEDLINE to identify potentially relevant literature investigating PPI requirement and dependency after TAVI. Study data, patients, and procedural characteristics were extracted. Odds ratio (OR) with $95 \%$ confidence intervals were extracted.

Results: Data from 23 studies were obtained that included 18,610 patients. The crude incidence of PPI after TAVI was $17 \%$ (range, $8.8 \%-32 \%$ ). PPI occurred at a median time of 3.2 days (range, $0-30$ days). Pacing dependency at 1-year was 47.5\% (range, $7 \%-89 \%$ ). Self-expandable prosthesis (pooled OR was 2.14 [1.153.96]) and baseline right bundle branch block (pooled OR was 2.01 [1.06-3.83]) showed 2-fold greater risk to maintain PPI dependency at 1 year after TAVI.

Conclusions: Although PPI represents a rather frequent event after TAVI, conduction disorders have a temporary nature in almost $50 \%$ of the cases with recovery and stabilization after discharge. Preoperative conduction abnormality and type of TAVI are associated with higher PPI dependency at short term. (JTCVS Open 2021;6:41-55)

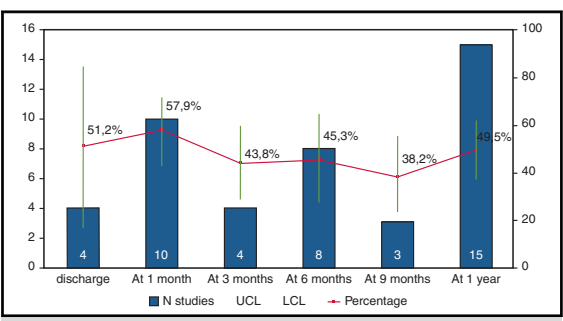

Rate of pacemaker dependency across the time after TAVI.

CENTRAL MESSAGE

Up to $50 \%$ of the patients with permanent pacemaker implantation following TAVI exhibit no pacemaker dependency at 1-year follow-up.

\section{PERSPECTIVE}

Better understanding of pacemaker dependency after TAVI should allow better oriented guidelines with respect to indications and timing of pacemaker implantation, as well as postpermanent pacemaker implantation management, based on the high recovery rate of effective native atrio/ ventricular conduction.

See Commentaries on pages 56 and 58.

(4) Supplemental material is available online.

From the ${ }^{\mathrm{a}}$ Department of Cardio-Thoracic Surgery, Heart and Vascular Centre, ${ }^{\mathrm{b}} \mathrm{De}-$ partment of Cardiology, and ${ }^{\mathrm{c}}$ Cardiovascular Research Institute Maastricht (CARIM), Maastricht University Medical Centre (MUMC+), Maastricht; and ${ }^{\mathrm{d}}$ Department of Cardiology, Radboud University Medical Center (Radboudumc), Nijmegen, The Netherlands.

Received for publication Feb 1, 2021; accepted for publication Feb 9, 2021; available ahead of print March 9, 2021.

Address for reprints: Justine M. Ravaux, MD, Department of Cardio-Thoracic Surgery, Heart \& Vascular Centre, Maastricht University Medical Centre (MUMC+), Cardiovascular Research Institute Maastricht (CARIM), Maastricht University, P. Debyelaan, 25, 6202 AZ Maastricht, The Netherlands (E-mail: justine.ravaux@ mumcl.nl or jmravaux@ hotmail.com).

2666-2736

Copyright (C) 2021 The Authors. Published by Elsevier Inc. on behalf of The American Association for Thoracic Surgery. This is an open access article under the CC BY-NCND license (http://creativecommons.org/licenses/by-nc-nd/4.0/).

https://doi.org/10.1016/j.xjon.2021.02.002
Transcatheter aortic valve implantation (TAVI) was first introduced in $2002^{1}$ as a less-invasive therapeutic option in patients with severe symptomatic aortic stenosis unfit for cardiac surgery. ${ }^{2}$ Nowadays, there is a trend to extend these procedures even to intermediate- and low-risk patients, making the frequency of TAVI procedures grow exponentially. ${ }^{3}$ However, complications, such as atrioventricular conduction disturbances requiring permanent pacemaker implantation (PPI), may diminish the benefit of these procedures: the incidence of PPI after TAVI, for instance, represents a rather frequent event after TAVI. ${ }^{4}$ Indeed, an 


$$
\begin{aligned}
& \text { Abbreviations and Acronyms } \\
& \begin{aligned}
\text { AF } & =\text { atrial fibrillation } \\
\text { BE } & =\text { balloon-expandable } \\
\text { CI } & =\text { confidence interval } \\
\text { OR } & =\text { odds ratio } \\
\text { PPI } & =\text { permanent pacemaker implantation } \\
\text { RBBB } & =\text { right bundle branch block } \\
\text { SE } & =\text { self-expandable } \\
\text { STS } & =\text { Society of Thoracic Surgeons } \\
\text { TAVI } & =\text { transcatheter aortic valve implantation }
\end{aligned}
\end{aligned}
$$

association between PPI and all-cause deaths and heart failure rehospitalizations at 1 year from TAVI has been recently shown. ${ }^{5}$ However, a certain percentage of atrioventricular conduction abnormalities after TAVI may resolve following PPI, even after a few days from implant. ${ }^{6}$ Presently, the current literature available on pacing dependency after TAVI is limited and based on studies with small patient samples (evidence level B) ${ }^{7,8}$ Therefore, we performed this systematic review to determine the incidence of PPI, the pacing dependency, and potential predictors for pacing-dependency at 1 year after TAVI procedures.

\section{METHODS}

\section{Research Strategy}

A broad, computerized literature search was performed to identify all relevant studies from PubMed, Embase, and MEDLINE databases. The PubMed database was searched entering the following key words: "Pacemaker, Artificial"[Mesh] OR pacemaker implantation AND "Transcatheter Aortic Valve Replacement"[Mesh] OR transcatheter aortic valve implantation. We restricted the research to English-language publications. Last access to the database was on April 25, 2020. The search was limited to studies in human recipients. A framework of the systematic review process is plotted in Figure 1, in accordance with the Preferred Reporting Items for Systematic Reviews and Meta-Analyses (PRISMA) guidelines. Because this study was a systematic review and meta-analysis based on published articles, ethical approval was waived by the institutional review board of the University Hospital of Maastricht.

\section{Eligibility Criteria and Study Selection}

Studies were included in the final analyses if patients were (1) $>18$ years; $(2)>250$ patients were included in the main analysis, to provide data interpretation of the most consistent clinical series; and (3) studies provided a definition of cardiac pacemaker dependency. Other studies following the same criteria, but having a smaller patient sample size $(<250$ patients), were included in a separated analysis (secondary analysis) and are provided in Appendix E1. Multiple publications from a single center were managed to include the last publication. If the same authors published more studies with the same series, the largest patient cohort was included. Studies were excluded if 1 of the following criteria was present: (1) presence of congenital pathology; (2) patients undergoing noncardiac surgery procedures or transcatheter procedure or heart transplantation; (3) no information provided about PPI; (4), publication before year 2002; or (5) outcomes not clearly reported or impossible to extract or calculate from the available results. Review, clinical update, and case reports were not taken into account. All potentially relevant studies were reviewed in detail to check their adhesion to the inclusion criteria. Title and abstracts of all retrieved paper were independently reviewed by 2 researchers (J.R. and M.D.M.) to identify studies fulfilling the inclusion criteria. Controversial findings were solved by the intervention of a third reviewer (R.L.). The quality of included studies was assessed using the Newcastle-Ottawa Scale for observational studies by 2 investigators independently (J.R. and M.D.M.).

\section{Data Extraction}

Microsoft Office Excel 2016 (Microsoft, Redmond, Wash) was used for data extraction that was performed independently by 2 researchers (K.V., L.V.). Year of publication, study design, sample size, age, Society of Thoracic Surgeons (STS) score, inclusion period, left ventricular ejection fraction, peripheral vascular disease, diabetes mellitus, valve type, follow-up, approach for TAVI, indications for PPI, timing of PPI (days), PPI rate, dependency definition, dependency follow-up (months), multivariable predictors of PPI, and PPI-related complications were extracted.

\section{Statistical Analysis}

The primary end point was 1-year pacing dependency, defined in different ways. Calculation of proportions of PM dependency at different time points was obtained using a meta-analytic approach by means of metaprop function of meta package in R (Foundation for Statistical Computing, Vienna, Austria). Odds ratio (OR) with $95 \%$ confidence intervals (CIs) were extracted. We calculated the $\mathrm{I}^{2}$ statistics $(0 \% \sim 100 \%)$ to explain the between-study heterogeneity, with $\mathrm{I}^{2} \leq 25 \%$ suggesting more homogeneity, $25 \%<\mathrm{I}^{2} \leq 75 \%$ suggesting moderate heterogeneity, and $\mathrm{I}^{2}>75 \%$ suggesting high heterogeneity. If the null hypothesis was rejected, a random effects model was used to calculate pooled effect estimates. If the null hypothesis was not rejected, a fixed-effects model was used to calculate pooled effect estimates; $95 \%$ CI was also reported. Forest plots were used to plot the effect size, either for each study or overall.

Publication bias was evaluated by graphical inspection of funnel plot; estimation of publication bias was performed with trim-and-fill method and quantified by means of Egger's linear regression test. On-leave out study analysis was performed as sensitivity analysis in case of moderate or high heterogeneity. Meta-regression was performed to test the influence of age, time of pacemaker implantation, and sample size. The software used for the analyses was R-studio (meta package), version 1.1.463 (2009-2018).

\section{RESULTS \\ Study Selection}

A total of 877 records were initially screened at the title and abstract level, with 801 papers fully reviewed for eligibility. There were no duplicate data. Ultimately, 23 studies were identified and provided data for the research analysis. ${ }^{9-31}$ An additional 30 studies are considered in Appendix E1 because of their limited sample size $(<250$ patients). The flowsheet for selection of included studies is represented in Figure 1. The Newcastle-Ottawa Scale confirmed a high-quality level for all studies included in the main analysis (Table E1).

\section{Study, Participants, and Procedural Characteristics}

Baseline characteristics of selected studies are represented in Table 1. Studies were published between 2014 and 2019, and patient recruitment occurred between January 2005 and February 2018; 26\% of them $(n=6)$ were prospective, ${ }^{9,16,18,27,29,31}$ and the remaining studies 


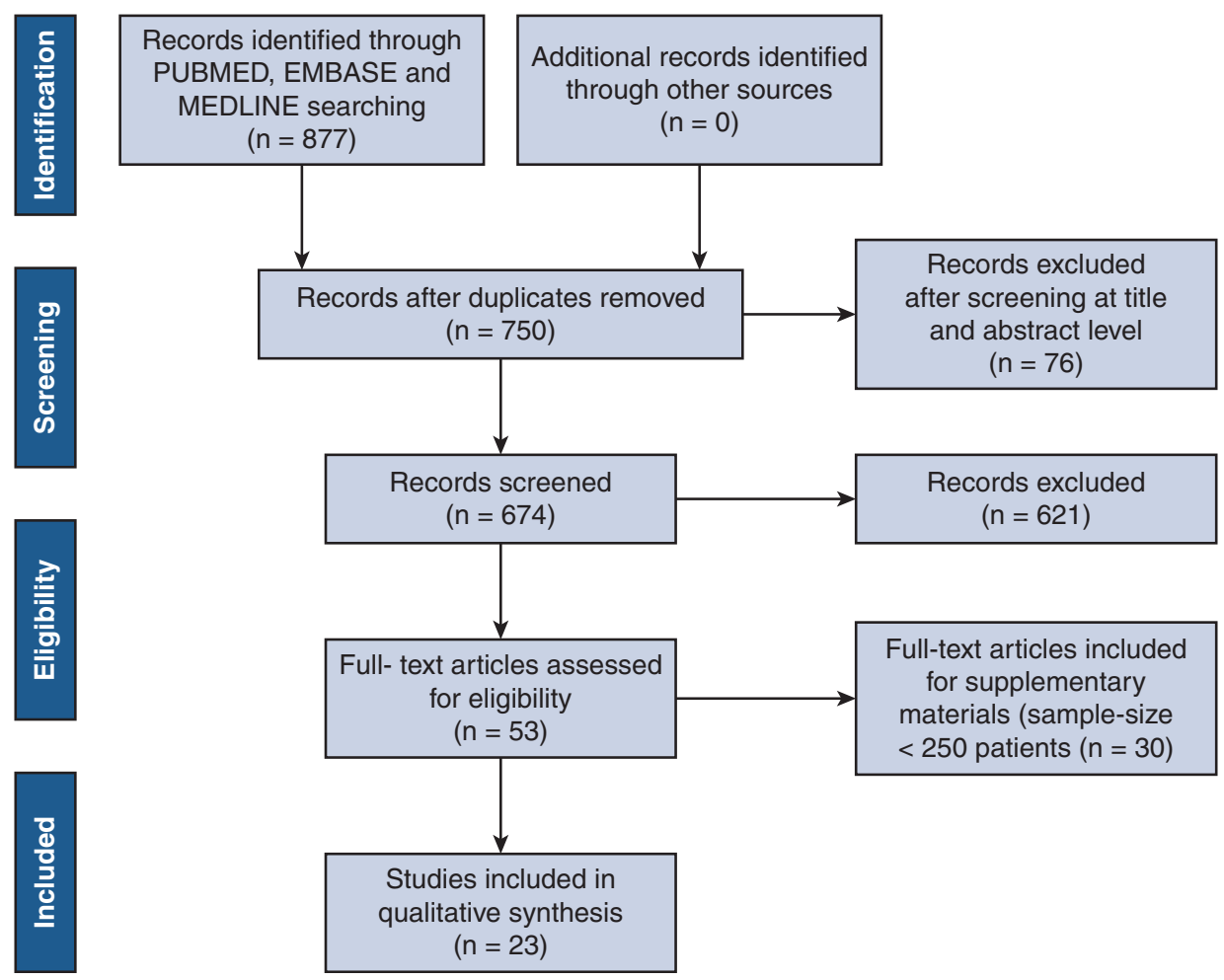

FIGURE 1. Study selection. Flow diagram of included studies based on the Preferred Reported Items for Systematic Reviews and Meta-Analysis (PRISMA).

were all retrospective observational. Mean duration of follow-up was 16 months.

In total, 18,610 patients were included in 23 studies from 79 centers. The crude incidence of PPI after TAVI procedure was $17 \%$, ranging from $8.8 \%{ }^{11}$ to $32 \%{ }^{14}$ Preprocedural risk was assessed by the STS score in the majority of the studies (11/23) and the mean STS score was $7.1(0.74-34)$. Mean age was 81.8 years (range, 43-102 years). The implanted device was a self-expandable (SE) valve in $38 \%$ of patients, a mechanically expandable valve in $13.5 \%$, and a balloon-expandable (BE) in $46 \%$ of the patient population. Others devices were used in $2.5 \%$ of the patients. Three studies used only BE valves, ${ }^{11,21,28}$ and 3 studies implanted solely SE valve. ${ }^{9,24,26}$ The transfemoral access route was preferred $(88.2 \%)$ over the other approaches (transapical $2.3 \%$; transsubclavian $0.9 \%$, and other approach used in $8.7 \%$ ). The mortality at 30 days was $9.6 \%$. The baseline characteristics of the studies including $<250$ patients are provided in Table E2.

\section{PPI Details}

The PPI details in studies including $>250$ patients are reported in Table 2. Data regarding PPI details in studies including $<250$ patients are described in Table E3. All studies provided indications for PPI, except in 4 series. ${ }^{20,24,25,30}$ The main indications for PPI were divided into 3 main categories of atrioventricular conduction disorders: atrioventricular block (second and third degree) in $82.7 \%$ of the patients, sick sinus syndrome in $2.7 \%$, severe bradycardia in $2.8 \%$, and others indications in $11.8 \%$ of the patients, respectively. The timing of PPI varied remarkably among studies, occurring at a median time of 3.2 days (range, 0-30 days) from TAVI procedure. Nine studies didn't provide information about the timing of PPI. ${ }^{9,18-21,24,25,27,29}$ The overall incidence of PPI reached $17 \%$ of the TAVI patients, ranging from $8.8 \%$ to $32 \%$ of the cases.

\section{Pacemaker Dependency}

There was a large heterogeneity in the assessment and definition of the pacemaker dependency at follow-up. Indeed, $43 \%$ of the studies $(10 / 23)^{10-12,14,16,18-20,25,28}$ reported a right ventricular pacing rhythm as indicator of the pacemaker dependency at follow-up, whereas the remaining studies provided a wide range of pacemaker dependency definitions and evaluation.

Of the selected 23 articles, the majority (15 studies $^{10-15,19,20,24-28,30,31}$ ) reported the 1-year pacemaker dependency (Figure 2). The pacemaker dependency rate varied across time (Figure 3); it was $51.2 \%(16.7 \%-84.6 \%, \mathrm{n}=4)$ already at discharge, $57.9 \%(43.1 \%-71.3 \%, \mathrm{n}=10)$ at 1 month, $45.3 \% \%(27.5 \%-64.5 \%, \mathrm{n}=8)$ at 6 months, and $49.5 \%(37.1 \%-61.9 \%, \mathrm{n}=15)$ at 1 year. 


\begin{tabular}{|c|c|c|c|c|c|c|c|c|c|c|c|c|c|}
\hline Study & Year & $\begin{array}{c}\text { Study } \\
\text { design (no. } \\
\text { centers) }\end{array}$ & $\begin{array}{c}\text { Sample } \\
\text { size }\end{array}$ & Age, $y$ & $\begin{array}{c}\text { STS } \\
\text { score, } \\
\%\end{array}$ & $\begin{array}{l}\text { Inclusion } \\
\text { period }\end{array}$ & $\begin{array}{c}\text { Left } \\
\text { ventricle } \\
\text { ejection } \\
\text { fraction, } \\
\%\end{array}$ & $\begin{array}{c}\text { Periphera } \\
\text { vascular } \\
\text { disease, } \\
\%\end{array}$ & $\begin{array}{l}\text { Diabetes } \\
\text { mellitus } \\
\%\end{array}$ & $\begin{array}{l}\text { Valve } \\
\text { type }\end{array}$ & $\begin{array}{c}\text { Follow- } \\
\text { up, } \\
\text { mo* }\end{array}$ & $\begin{array}{c}\text { Approach } \\
\text { for TAVI }\end{array}$ & $\begin{array}{c}\text { Mortality } \\
\text { at } 30 \mathrm{~d}\end{array}$ \\
\hline $\begin{array}{l}\text { Bjerre } \\
\text { Thygesen } \\
\text { et } \mathrm{al}^{9}\end{array}$ & 2014 & $\begin{array}{c}\text { Prospective } \\
\text { (1) }\end{array}$ & 258 & na & na & na & na & na & na & $100 \% \mathrm{SE}$ & na & na & na \\
\hline $\begin{array}{l}\text { Urena } \\
{\text { et } \mathrm{al}^{10}}^{10}\end{array}$ & 2014 & $\begin{array}{c}\text { Retrospective } \\
\text { (8) }\end{array}$ & 1556 & 80.5 & 7.5 & $\begin{array}{c}\text { January } 2005 \text { to } \\
\text { February } 2013\end{array}$ & 55.5 & na & 31.1 & $\begin{array}{l}55.1 \% \mathrm{BE} \\
44.9 \% \mathrm{SE}\end{array}$ & 22 & na & $7 \%$ \\
\hline $\begin{array}{l}\text { Nazif } \\
\quad \text { et } \mathrm{al}^{11}\end{array}$ & 2015 & $\begin{array}{c}\text { Retrospective } \\
\text { (21) }\end{array}$ & 1973 & 84.5 & 11.4 & $\begin{array}{c}\text { May } 2007 \text { to } \\
\text { September } 2011\end{array}$ & 53.7 & 42.4 & 36 & $100 \% \mathrm{BE}$ & 12 & na & $6.6 \%$ \\
\hline $\begin{array}{r}\text { Van Gils } \\
\text { et } \mathrm{al}^{12}\end{array}$ & 2017 & $\begin{array}{l}\text { Retrospective } \\
\text { (4) }\end{array}$ & 306 & 83 & 6.3 & $\begin{array}{l}\text { May } 2008 \text { to } \\
\text { February } 2016\end{array}$ & na & 22 & 30 & $\begin{array}{l}38.2 \% \mathrm{SE} \\
34.7 \% \mathrm{SE} \\
27.1 \% \mathrm{ME}\end{array}$ & 12 & na & $7 \%$ \\
\hline $\begin{array}{l}\text { Raelson } \\
\text { et }^{13} l^{13}\end{array}$ & 2017 & $\begin{array}{c}\text { Retrospective } \\
\text { (1) }\end{array}$ & 578 & 85.5 & na & $\begin{array}{l}\text { March } 2009 \text { to } \\
\text { December } 2014\end{array}$ & na & na & na & $\begin{array}{l}21 \% \mathrm{SE} \\
79 \% \mathrm{BE}\end{array}$ & 1 & na & na \\
\hline $\begin{array}{l}\text { Dumonteil } \\
\text { et } \mathrm{al}^{14}\end{array}$ & 2017 & $\begin{array}{l}\text { Retrospective } \\
\text { (14) }\end{array}$ & 250 & 84 & 6.3 & $\begin{array}{l}\text { October } 2012 \\
\text { to May } 2014\end{array}$ & 58.3 & na & 22.5 & $100 \% \mathrm{ME}$ & 12 & $\begin{array}{c}100 \% \\
\text { Transfemoral }\end{array}$ & $4 \%$ \\
\hline $\begin{array}{l}\text { Kaplan } \\
\text { et } \mathrm{al}^{15}\end{array}$ & 2019 & $\begin{array}{c}\text { Retrospective } \\
\text { (1) }\end{array}$ & 594 & 81.6 & na & $\begin{array}{l}\text { January } 2011 \text { to } \\
\text { December } 2017\end{array}$ & na & na & na & na & 12 & na & na \\
\hline $\begin{array}{l}\text { Chamandi } \\
\text { et } \mathrm{al}^{16}\end{array}$ & 2018 & $\begin{array}{c}\text { Prospective } \\
\text { (9) }\end{array}$ & 1692 & 81.5 & 10.9 & $\begin{array}{l}\text { May } 2009 \text { to } \\
\text { February } 2015\end{array}$ & na & na & 33.1 & $\begin{array}{l}50.3 \% \mathrm{BE} \\
49.7 \% \mathrm{SE}\end{array}$ & 48 & na & $42.3 \%$ \\
\hline $\begin{array}{l}\text { Gaede } \\
{\text { et } \mathrm{al}^{17}}^{17}\end{array}$ & 2018 & $\begin{array}{c}\text { Retrospective } \\
\text { (1) }\end{array}$ & 1025 & 81.9 & na & $2010-2015$ & na & 21.1 & 33.3 & na & 2.4 & na & na \\
\hline 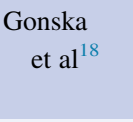 & 2018 & $\begin{array}{l}\text { Prospective } \\
\text { (1) }\end{array}$ & 612 & 80.4 & 6.5 & $\begin{array}{l}\text { February } 2014 \text { to } \\
\text { September } 2016\end{array}$ & 57.5 & na & 29.9 & $\begin{array}{c}58.8 \% \mathrm{BE} \\
36.7 \% \mathrm{ME} \\
4.4 \% \mathrm{SE}\end{array}$ & 12 & na & $1.3 \%$ \\
\hline $\begin{array}{r}\text { Marzahn } \\
\text { et al }^{19}\end{array}$ & 2018 & $\begin{array}{l}\text { Retrospective } \\
\text { (1) }\end{array}$ & 856 & 80.5 & na & $\begin{array}{c}\text { July } 2008 \text { to } \\
\text { May } 2015\end{array}$ & 57.5 & na & 38 & $\begin{array}{l}37.4 \% \mathrm{SE} \\
57.8 \% \mathrm{BE} \\
4.8 \% \mathrm{ME}\end{array}$ & 12 & $\begin{array}{c}100 \% \\
\text { Transfemoral }\end{array}$ & na \\
\hline $\begin{array}{r}\text { Nadeem } \\
\text { et }^{a^{20}}\end{array}$ & 2018 & $\begin{array}{l}\text { Retrospective } \\
\text { (1) }\end{array}$ & 672 & 81.4 & 7.4 & 2011-2017 & 53.8 & 21.5 & 41 & na & 12 & na & na \\
\hline $\begin{array}{l}\text { Campelo- } \\
\text { Parada } \\
\text { et } \mathrm{al}^{21}\end{array}$ & 2018 & $\begin{array}{l}\text { Retrospective } \\
\text { (2) }\end{array}$ & 347 & na & na & $\begin{array}{l}\text { May } 2010 \text { to } \\
\text { December } 2015\end{array}$ & 54.6 & na & 37.3 & $100 \% \mathrm{BE}$ & 1 & $\begin{array}{c}77.8 \% \\
\text { Transfemoral }\end{array}$ & na \\
\hline $\begin{array}{l}\text { Mirolo } \\
\text { et } \mathrm{al}^{22}\end{array}$ & 2018 & $\begin{array}{c}\text { Retrospective } \\
\text { (1) }\end{array}$ & 936 & na & na & $\begin{array}{l}\text { October } 2009 \text { to } \\
\text { January } 2017\end{array}$ & na & na & na & $\begin{array}{l}95 \% \mathrm{BE} \\
5 \% \mathrm{SE}\end{array}$ & 2.3 & na & na \\
\hline $\begin{array}{r}\text { Van Gils } \\
\text { et } \mathrm{al}^{23}\end{array}$ & 2018 & $\begin{array}{l}\text { Retrospective } \\
\text { (1) }\end{array}$ & 291 & 79 & na & $\begin{array}{l}\text { January } 2012 \text { to } \\
\text { December } 2015\end{array}$ & na & na & 34 & $\begin{array}{r}42 \% \mathrm{SE} \\
51.5 \% \mathrm{BE} \\
6.5 \% \mathrm{ME}\end{array}$ & 12 & $\begin{array}{c}94 \% \\
\text { transfemoral } \\
6 \% \\
\text { transsubclavian }\end{array}$ & $5 \%$ \\
\hline $\begin{array}{l}\text { Takahashi } \\
\text { et }^{2} \mathrm{al}^{24}\end{array}$ & 2018 & $\begin{array}{c}\text { Retrospective } \\
\text { (4) }\end{array}$ & 1621 & 84.3 & na & $\begin{array}{l}\text { January } 2010 \text { to } \\
\text { December } 2014\end{array}$ & na & na & na & $72.5 \%$ SE & 13 & na & na \\
\hline $\begin{array}{l}\text { Chan } \\
\text { et } \mathrm{al}^{25}\end{array}$ & 2018 & $\begin{array}{c}\text { Retrospective } \\
\text { (2) }\end{array}$ & 913 & 81.6 & na & $\begin{array}{l}\text { January } 2012 \text { to } \\
\text { December } 2017\end{array}$ & na & na & na & na & 12 & na & na \\
\hline $\begin{array}{c}\text { Ghannam } \\
\text { et } \mathrm{al}^{26}\end{array}$ & 2019 & $\begin{array}{c}\text { Retrospective } \\
\text { (1) }\end{array}$ & 573 & 79.8 & 6.4 & $\begin{array}{l}\text { January } 2012 \text { to } \\
\text { September } 2017\end{array}$ & 57.2 & 45.8 & 37.8 & $100 \% \mathrm{SE}$ & 17.1 & na & na \\
\hline 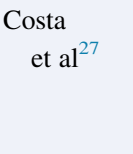 & 2019 & $\begin{array}{c}\text { Prospective } \\
\text { (1) }\end{array}$ & 1116 & 82 & 4.4 & $\begin{array}{l}\text { June } 2007 \text { to } \\
\text { February } 2018\end{array}$ & 53.3 & na & 28.9 & $\begin{array}{c}61.8 \% \mathrm{SE} \\
27.2 \% \mathrm{BE} \\
0.5 \% \mathrm{ME} \\
0.5 \% \text { Others }\end{array}$ & 72 & $\begin{array}{l}\quad 97 \% \\
\text { transfemoral } \\
3 \% \text { others }\end{array}$ & $3.9 \%$ \\
\hline
\end{tabular}




\begin{tabular}{|c|c|c|c|c|c|c|c|c|c|c|c|c|c|}
\hline Study & Year & $\begin{array}{c}\text { Study } \\
\text { design (no. } \\
\text { centers) }\end{array}$ & $\begin{array}{c}\text { Sample } \\
\text { size }\end{array}$ & Age, $\mathbf{y}$ & $\begin{array}{c}\text { STS } \\
\text { score, } \\
\%\end{array}$ & $\begin{array}{c}\text { Inclusion } \\
\text { period }\end{array}$ & $\begin{array}{c}\text { Left } \\
\text { ventricle } \\
\text { ejection } \\
\text { fraction, } \\
\%\end{array}$ & $\begin{array}{l}\text { Periphera } \\
\text { vascular } \\
\text { disease, } \\
\%\end{array}$ & $\begin{array}{c}\text { Diabetes } \\
\text { mellitus, } \\
\%\end{array}$ & $\begin{array}{l}\text { Valve } \\
\text { type }\end{array}$ & $\begin{array}{c}\text { Follow- } \\
\text { up, } \\
\text { mo* }\end{array}$ & $\begin{array}{c}\text { Approach } \\
\text { for TAVI }\end{array}$ & $\begin{array}{c}\text { Mortality } \\
\text { at } 30 \mathrm{~d}\end{array}$ \\
\hline $\begin{array}{l}\text { Dolci } \\
\text { et } \mathrm{al}^{28}\end{array}$ & 2019 & $\begin{array}{l}\text { Retrospective } \\
\text { (1) }\end{array}$ & 266 & 80 & na & $\begin{array}{c}\text { February } 2014 \\
\text { to February } \\
2018\end{array}$ & 53 & 22 & 28 & $100 \% \mathrm{BE}$ & 12 & $\begin{array}{c}84 \% \\
\text { transfemoral } \\
16 \% \\
\text { transapical }\end{array}$ & na \\
\hline 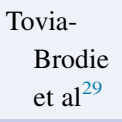 & 2019 & $\begin{array}{c}\text { Prospective } \\
\text { (1) }\end{array}$ & 795 & 82.5 & na & $\begin{array}{c}\text { April } 2012 \text { to } \\
\text { December } 2016\end{array}$ & na & na & na & na & 28.2 & na & na \\
\hline 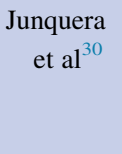 & 2019 & $\begin{array}{l}\text { Retrospective } \\
\text { (2) }\end{array}$ & 676 & 82 & 5 & $\begin{array}{l}\text { May } 2007 \text { to } \\
\text { March } 2017\end{array}$ & 57.4 & na & 31.2 & $\begin{array}{l}60.5 \% \mathrm{BE} \\
35.2 \% \mathrm{SE} \\
0.7 \% \mathrm{ME} \\
0.3 \% \text { others }\end{array}$ & 12 & $\begin{array}{c}64.8 \% \\
\text { transfemoral }\end{array}$ & na \\
\hline $\begin{array}{l}\text { Meduri } \\
{\text { et } \mathrm{al}^{31}}^{31}\end{array}$ & 2019 & $\begin{array}{l}\text { Prospective } \\
\text { (1) }\end{array}$ & 704 & 82.5 & 6.6 & na & na & 28.2 & 30.9 & $\begin{array}{l}34 \% \mathrm{SE} \\
66 \% \mathrm{ME}\end{array}$ & 12 & na & na \\
\hline Total & na & 79 & 18,610 & $\begin{array}{c}81.8 \\
(43-102)\end{array}$ & $\begin{array}{c}7.1 \\
0.74-34)\end{array}$ & $\begin{array}{c}\text { January } 2005 \text { to } \\
\text { February } 2018\end{array}$ & 55.6 & 29 & $\begin{array}{r}32.6 \\
1 \\
\text { o }\end{array}$ & $\begin{array}{c}\text { SE } 38 \% \\
\text { BE } 46 \% \\
\text { ME } 13.5 \% \\
\text { others } 2.5 \%\end{array}$ & 16 & $\begin{array}{c}88.2 \% \\
\text { transfemoral } \\
2.3 \% \\
\text { transapical } \\
0.9 \% \\
\text { transsubclavian } \\
\text { Others } 8.7 \%\end{array}$ & $9.6 \%$ \\
\hline
\end{tabular}

Values are n (\%). STS score, Society of Thoracic Surgeons Risk Score; TAVI, transaortic valve implantation; $n a$, not available; SE, self-expandable; $B E$, balloon-expandable; $M E$, mechanically expandable. *Follow-up is reported as mean or median as given by the authors.

\section{Influence of Baseline Right Bundle Branch Block (RBBB) and Atrial Fibrillation (AF) on 1-Year Pacemaker Dependency}

In 6 studies, ${ }^{13,15,20,25,27,31}$ among 531 patients undergoing pacemaker interrogation at 1 year, pacemaker dependency was present from $22 \%$ to $54 \%$ of the patients, and the difference between patients with and without baseline RBBB could be analyzed. The pooled OR was $1.76(95 \% \mathrm{CI}$, 1.06-2.93). There was low heterogeneity $\left(\mathrm{I}^{2}=14 \%\right)$ among the studies (Figure 4), nor was there publication bias (Figure E1). The Egger's test was not significant $(P=.90)$. Leave-one-out study analysis (Table E4), as shown in the paper by Chan and colleagues ${ }^{25}$ influenced the pooled analysis, since leaving it in, the pooled estimates was not significant anymore. The association between preimplant RBBB and 1-year pacemaker dependency was not influenced by age (r $=0.0917, P=.6050)$, time of PPI $(\mathrm{r}=0.0918$, $P=.9222)$, or sample size $(\mathrm{r}=0.0920, P=.9245)$.

The impact of baseline AF could be investigated in 4 studies $^{13,15,20,25}$ and revealed no effect on 1-year pacemaker dependency (pooled OR, 1.71; 95\% CI, 0.83-3.53) (Figure 5). Again, there was neither heterogeneity among the studies nor publication bias (Figure E2) The Egger's test was not significant $(P=.79)$. On-leave out study analysis (Table E4) confirmed as AF had no impact on pooled analysis.

\section{Type of Implanted Prosthesis on 1-Year Pacemaker Dependency}

The comparison between SE and BE protheses in terms of 1-year pacemaker dependency could be evaluated in 6studies (796 patients). ${ }^{10,12,13,15,20,24}$ Patients who received SE prostheses had 2-fold greater risk for pacemaker dependency 1 year after TAVI (Figure 6). moderate heterogeneity was found $\left(\mathrm{I}^{2}=43 \%\right)$. No publication bias was identified (Figure E3). The Egger's test was not significant $(P=.5658)$. Leave-one-out study analysis (Table E4) showed as the paper by Urena and colleagues ${ }^{10}$ influenced the pooled analysis, since leaving it, the pooled estimates was not significant anymore. The association between preimplant SE and 1-year pacemaker dependency was not influenced by age $(\mathrm{r}=0.1265, P=.5012)$, time of PPI (r $=-1.1506, P=.0 .6262)$, or sample size $(\mathrm{r}=0.1123$, $P=.9812$ ). The pacemaker dependency rate was significantly greater in those studies including more than $50 \%$ of SE prostheses (Figure E4).

\section{Third-Degree Atrioventricular Block and 1-Year Pacemaker Dependency}

One-year pacemaker dependency rate was not significantly different between study where pacemaker was implanted due to third-degree atrioventricular block in a rate ranging from $70 \%-79 \%(41.4 \% ; 26.1 \%-58.5 \%)$, 


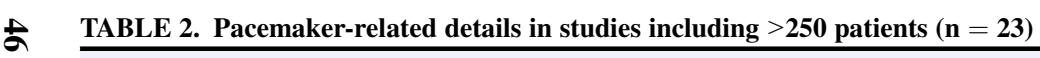

\begin{tabular}{|c|c|c|c|c|c|c|c|c|c|}
\hline Study & $\begin{array}{l}\text { Indications } \\
\text { for PPI }\end{array}$ & $\begin{array}{c}\text { Timing } \\
\text { of PPI, } \mathbf{d}^{*}\end{array}$ & $\begin{array}{l}\text { PPI } \\
\text { rate }\end{array}$ & $\begin{array}{c}\text { Dependency } \\
\text { definition }\end{array}$ & $\begin{array}{l}\text { Dependency } \\
\text { rate }\end{array}$ & $\begin{array}{l}\text { Dependency } \\
\text { follow-up, } \\
\text { mo* }\end{array}$ & $\begin{array}{c}\text { Multivariable } \\
\text { predictors } \\
\text { of PPI }\end{array}$ & Association & $\begin{array}{c}\text { PPI-related } \\
\text { complications }\end{array}$ \\
\hline 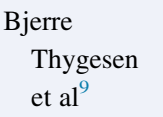 & $100 \%$ AVB & na & $27.4 \%$ & $\begin{array}{l}\text { Resolution of } \\
\text { conduction } \\
\text { abnormalities }\end{array}$ & $50 \%$ & na & na & na & na \\
\hline${\text { Urena et } \mathrm{al}^{10}}^{10}$ & $\begin{array}{l}75.3 \% \text { AVB } \\
7.1 \% \text { SSS } \\
7.9 \% \\
\text { bradycardia } \\
9.6 \% \text { others }\end{array}$ & 3 & $15.4 \%$ & $\begin{array}{l}\text { "paced rhythm" } \\
\text { reported }\end{array}$ & $66.9 \%$ & 12 & na & $\begin{array}{l}\text { PPI protective factor } \\
\text { for the occurrence of } \\
\text { unexpected (sudden } \\
\text { or unknown) death } \\
\text { - Negative effect on } \\
\text { left ventricular } \\
\text { function over time }\end{array}$ & na \\
\hline Nazif et al ${ }^{11}$ & $\begin{array}{l}79 \% \text { AVB } \\
17.3 \% \text { SSS }\end{array}$ & 3 & $8.8 \%$ & $\begin{array}{l}\text { "ventricular } \\
\text { pacing" } \\
\text { reported }\end{array}$ & $50.5 \%$ & 12 & $\begin{array}{l}\text { - Pre-existing } \\
\text { RBBB } \\
\text { - Prosthesis to left } \\
\text { ventricle outflow } \\
\text { tract diameter ratio } \\
\text { - Left ventricle -end } \\
\text { diastolic diameter }\end{array}$ & $\begin{array}{l}\text { - Longer duration } \\
\text { of hospitalization } \\
\text { - Greater rates of } \\
\text { repeat } \\
\text { hospitalization } \\
\text { and mortality or } \\
\text { repeat } \\
\text { hospitalization } \\
\text { at } 1 \mathrm{y}\end{array}$ & na \\
\hline $\begin{array}{l}\text { Van Gils } \\
\text { et al }^{12}\end{array}$ & $\begin{array}{l}99 \% \text { AVB } \\
1 \% \text { SSS }\end{array}$ & 2 & $41 \%$ & $\begin{array}{l}\% \text { ventricular } \\
\text { pacing rhythm } \\
\text { reported }\end{array}$ & $89 \%$ & 12 & $\begin{array}{l}\text { - LOTUS valve } \\
\text { - Greater BMI before } \\
\text { TAVI }\end{array}$ & $\begin{array}{l}\text { RBBB at baseline } \\
\text { associated with } \\
\text { greater PPI }\end{array}$ & na \\
\hline $\begin{array}{l}\text { Raelson } \\
\text { et al }^{13}\end{array}$ & $82 \%$ AVB & 3 & $9 \%$ & $\begin{array}{l}\text { No intrinsic } \\
\text { ventricular } \\
\text { activity } \\
\text { during pacing } \\
\text { at } 30 \mathrm{bpm}\end{array}$ & $39 \%$ & 1 & na & na & na \\
\hline $\begin{array}{l}\text { Dumonteil } \\
{\text { et } \mathrm{al}^{14}}^{14}\end{array}$ & $\begin{array}{l}88.9 \% \text { AVB } \\
5.9 \% \text { others }\end{array}$ & 3 & $32 \%$ & $\begin{array}{l}\text { "paced rhythm” } \\
\text { reported }\end{array}$ & $55.4 \%$ & 12 & $\begin{array}{l}\text { - Baseline RBBB } \\
\text { - Left ventricle outflow } \\
\text { tract overstretch }>10 \%\end{array}$ & $\begin{array}{l}\text { - Trend lower PPI } \\
\text { rate at } 30 \mathrm{~d} \text { with } \\
\text { shallower }(\leq 5 \mathrm{~mm}) \\
\text { implant depth }\end{array}$ & na \\
\hline
\end{tabular}




\begin{tabular}{|c|c|c|c|c|c|c|c|c|c|}
\hline Study & $\begin{array}{l}\text { Indications } \\
\text { for PPI }\end{array}$ & $\begin{array}{c}\text { Timing } \\
\text { of PPI, } \mathbf{d}^{*}\end{array}$ & $\begin{array}{l}\text { PPI } \\
\text { rate }\end{array}$ & $\begin{array}{c}\text { Dependency } \\
\text { definition }\end{array}$ & $\begin{array}{l}\text { Dependency } \\
\text { rate }\end{array}$ & $\begin{array}{c}\text { Dependency } \\
\text { follow-up, } \\
\text { mo* }\end{array}$ & $\begin{array}{c}\text { Multivariable } \\
\text { predictors } \\
\text { of PPI }\end{array}$ & Association & $\begin{array}{l}\text { PPI-related } \\
\text { complications }\end{array}$ \\
\hline Kaplan et al $^{15}$ & $\begin{array}{l}79 \% \text { AVB } \\
21 \% \text { others }\end{array}$ & 2,5 & $13.1 \%$ & $\begin{array}{l}\text { High-grade } \\
\text { AVB with a } \\
\text { ventricular } \\
\text { escape rate of } \\
\text { less than } \\
40 \text { beats/min } \\
\text { on device } \\
\text { interrogation }\end{array}$ & $21.9 \%$ & 12 & na & $\begin{array}{l}\text { Use of SE valves } \\
\text { and postballoon } \\
\text { dilatation associated } \\
\text { with markedly } \\
\text { increased risk of } \\
\text { PPM dependency }\end{array}$ & na \\
\hline $\begin{array}{l}\text { Chamandi } \\
\text { et } \mathrm{al}^{16}\end{array}$ & $\begin{array}{l}76.7 \% \text { AVB } \\
5.6 \% \text { SSS } \\
3.1 \% \\
\text { Bradycardia } \\
14.6 \% \text { others }\end{array}$ & 2 & $19.8 \%$ & $\begin{array}{l}100 \% \\
\text { right } \\
\text { ventricular } \\
\text { pacing }\end{array}$ & $27.4 \%$ & 48 & na & $\begin{array}{l}\text { PPI greater rates } \\
\text { of rehospitalization } \\
\text { due to heart failure } \\
\text { and combined end } \\
\text { point of mortality } \\
\text { or heart failure } \\
\text { rehospitalization } \\
\text { - PPI lesser } \\
\text { improvement in } \\
\text { left ventricle } \\
\text { ejection fraction } \\
\text { over time, } \\
\text { particularly if } \\
\text { reduced before } \\
\text { TAVI }\end{array}$ & na \\
\hline Gaede et $\mathrm{al}^{17}$ & $\begin{array}{l}90 \% \text { AVB } \\
8 \% \text { SSS } \\
2 \% \\
\quad \text { Bradycardia }\end{array}$ & 4 & $14.7 \%$ & $\begin{array}{l}\text { Ventricular } \\
\text { pacing }>95 \%\end{array}$ & $29.5 \%$ & 2.4 & $\begin{array}{l}\text { - Pre-existing } \\
\text { RBBB } \\
\text { - CoreValve } \\
\text { prosthesis }\end{array}$ & $\begin{array}{l}\text { Predictors } \\
\text { of lack of } \\
\text { recovery } \\
\text { of AVB } \\
\text { - Previous RBBB } \\
\text { - Greater mean } \\
\text { aortic valve } \\
\text { gradient } \\
\text { - Postdilatation } \\
\text { of the prosthesis }\end{array}$ & na \\
\hline $\begin{array}{l}\text { Gonska } \\
\text { et }^{18}{ }^{18}\end{array}$ & $\begin{array}{l}85 . \% \text { AVB } \\
10.1 \% \\
\text { Bradycardia } \\
4.8 \% \text { others }\end{array}$ & na & $24.4 \%$ & $\begin{array}{l}\text { "ventricular } \\
\text { pacing" } \\
\text { reported }\end{array}$ & $30.9 \%$ & 1 & na & $\begin{array}{l}\text { - PPI without } \\
\text { significant } \\
\text { impact on survival } \\
\text { or combined end } \\
\text { point of major } \\
\text { adverse events } \\
\text { within } 1 \text { y }\end{array}$ & $\begin{array}{l}1.8 \% \text { reoperation } \\
\text { due to lead } \\
\text { dislocation } \\
2.4 \% \text { hematomas/ } \\
\text { bleeding at the } \\
\text { site of the } \\
\text { pacemaker }\end{array}$ \\
\hline
\end{tabular}




\begin{tabular}{|c|c|c|c|c|c|c|c|c|c|}
\hline Study & $\begin{array}{l}\text { Indications } \\
\text { for PPI }\end{array}$ & $\begin{array}{c}\text { Timing } \\
\text { of PPI, } \mathbf{d}^{*}\end{array}$ & $\begin{array}{l}\text { PPI } \\
\text { rate }\end{array}$ & $\begin{array}{l}\text { Dependency } \\
\text { definition }\end{array}$ & $\begin{array}{l}\text { Dependency } \\
\text { rate }\end{array}$ & $\begin{array}{c}\text { Dependency } \\
\text { follow-up, } \\
\text { mo* }\end{array}$ & $\begin{array}{c}\text { Multivariable } \\
\text { predictors } \\
\text { of PPI }\end{array}$ & Association & $\begin{array}{c}\text { PPI-related } \\
\text { complications }\end{array}$ \\
\hline $\begin{array}{r}\text { Marzahn } \\
\text { et al }{ }^{19}\end{array}$ & $\begin{array}{l}89 \% \text { AVB } \\
5.5 \% \\
\quad \text { Bradycardia } \\
4.1 \% \text { SSS } \\
1.4 \% \text { others }\end{array}$ & na & $16.9 \%$ & $\begin{array}{l}\text { "right } \\
\text { ventricular } \\
\text { pacing \%" } \\
\text { reported }\end{array}$ & $55 \%$ & 12 & na & na & na \\
\hline $\begin{array}{l}\text { Nadeem } \\
{\text { et } \mathrm{al}^{20}}^{20}\end{array}$ & na & na & $21.7 \%$ & $\begin{array}{l}\text { "right } \\
\text { ventricular } \\
\text { pacing \%" } \\
\text { reported }\end{array}$ & $45.5 \%$ & 12 & na & $\begin{array}{l}\text { PPI more likely } \\
\text { to have heart } \\
\text { failure admissions } \\
\text { - PPI trend toward } \\
\text { increased mortality }\end{array}$ & na \\
\hline $\begin{array}{c}\text { Campelo- } \\
\text { Parada } \\
\text { et } \mathrm{al}^{21}\end{array}$ & $\begin{array}{l}84.3 \% \text { AVB } \\
9.3 \% \\
\text { Bradycardia } \\
\text { Others } 6.2 \%\end{array}$ & na & $9.2 \%$ & $\begin{array}{l}\text { Ventricular } \\
\text { pacing }>1 \% \\
\text { at } 1 \mathrm{mo}= \\
\text { AVB } \\
\text { resolution }\end{array}$ & $67.2 \%$ & 1 & na & $\begin{array}{l}\text { BAV associated } \\
\text { with increased risk } \\
\text { of conduction } \\
\text { abnormalities } \\
\text { persistence }\end{array}$ & na \\
\hline $\begin{array}{l}\text { Mirolo } \\
\text { et }^{2 l^{22}}\end{array}$ & $\begin{array}{l}68.8 \% \text { AVB } \\
30 \% \text { others }\end{array}$ & 2,5 & $9.3 \%$ & $\begin{array}{l}\text { Ventricular } \\
\text { pacing } \\
\quad \geq 1 \% \\
\quad=\text { significant }\end{array}$ & $75 \%$ & 2.9 & na & na & $\begin{array}{l}1.25 \% \\
\text { endocarditis } \\
\text { lead leading } \\
\text { to pacemaker } \\
\text { explanation } \\
2.5 \% \text { partial left } \\
\text { pneumothorax } \\
\text { secondary to } \\
\text { subclavian } \\
\text { vein puncture } \\
1.25 \% \\
\text { ventricular } \\
\text { lead } \\
\text { deplacement }\end{array}$ \\
\hline $\begin{array}{r}\text { Van Gils } \\
\text { et } \mathrm{al}^{23}\end{array}$ & $\begin{array}{l}96 \% \text { AVB } \\
5 \% \text { SSS }\end{array}$ & 5 & $9.3 \%$ & $\begin{array}{l}\text { Less than } 20 \% \\
\text { ventricular } \\
\text { pacing over } \\
6 \text { mo' } \\
\text { follow-up }\end{array}$ & $25 \%$ & 6 & na & na & na \\
\hline
\end{tabular}


TABLE 2. Continued

\begin{tabular}{|c|c|c|c|c|c|c|c|c|c|}
\hline Study & $\begin{array}{l}\text { Indications } \\
\text { for PPI }\end{array}$ & $\begin{array}{c}\text { Timing } \\
\text { of PPI, } \mathbf{d}^{*}\end{array}$ & $\begin{array}{l}\text { PPI } \\
\text { rate }\end{array}$ & $\begin{array}{l}\text { Dependency } \\
\text { definition }\end{array}$ & $\begin{array}{l}\text { Dependency } \\
\text { rate }\end{array}$ & $\begin{array}{l}\text { Dependency } \\
\text { follow-up, } \\
\text { mo* }\end{array}$ & $\begin{array}{c}\text { Multivariable } \\
\text { predictors } \\
\text { of PPI }\end{array}$ & Association & $\begin{array}{c}\text { PPI-related } \\
\text { complications }\end{array}$ \\
\hline $\begin{array}{c}\text { Takahashi } \\
\text { et }^{2}{ }^{24}\end{array}$ & na & na & $16.4 \%$ & $\begin{array}{l}\text { Absence, } \\
\text { inadequate } \\
\text { intrinsic } \\
\text { ventricular } \\
\text { rhythm, or } \\
\text { ventricular } \\
\text { pacing }>95 \% \\
\text { in pacemaker } \\
\text { interrogation } \\
\text { during } \\
\text { follow-up } \\
\text { (PPM on VVI } \\
\text { 30/min) }\end{array}$ & $52.8 \%$ & 13 & na & $\begin{array}{l}\text { DDD mode and SE } \\
\text { valves use associated } \\
\text { with pacemaker } \\
\text { dependency }\end{array}$ & na \\
\hline $\begin{array}{l}\text { Chan } \\
{\text { et } \mathrm{al}^{25}}^{25}\end{array}$ & na & na & $13.1 \%$ & $\begin{array}{l}\text { Ventricular } \\
\text { pacing } \\
\text { reported }\end{array}$ & $59 \%$ & 12 & na & na & $\begin{array}{l}1.6 \% \text { atrial } \\
\text { lead } \\
\text { dislodgement } \\
6 \% \text { ventricular } \\
\text { lead } \\
\text { dislodgement }\end{array}$ \\
\hline 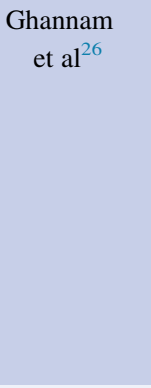 & $100 \%$ AVB & 2,4 & $14 \%$ & $\begin{array}{l}\text { No recovery of } \\
\text { AV nodal } \\
\text { conduction if } \\
\mathrm{CHB} \text {, high-grade } \\
\mathrm{AVB} \text {, or native } \\
\text { ventricular rate } \\
<50 \text { beats } / \text { min } \\
\text { in absence of } \\
\text { normal } \mathrm{AV} \\
\text { conduction }\end{array}$ & $50 \%$ & 12 & na & $\begin{array}{l}\text { - Larger aortic annulus } \\
\text { less likely to recover } \\
\text { conduction }\end{array}$ & $\begin{array}{l}1.2 \% \text { ( } 1 \text { patient } \\
\text { with right } \\
\text { ventricular } \\
\text { lead fracture) }\end{array}$ \\
\hline Costa et $\mathrm{al}^{27}$ & $\begin{array}{l}84.8 \% \text { AVB } \\
4.1 \% \text { SSS } \\
11 \% \text { Others }\end{array}$ & na & $13 \%$ & $\begin{array}{l}\text { Absence of an } \\
\text { escape or } \\
\text { intrinsic } \\
\text { rhythm for } 30 \mathrm{~s} \\
\text { during } \\
\text { temporary } \\
\text { back-up pacing } \\
\text { at a rate of } \\
30 \mathrm{bpm}\end{array}$ & $33.3 \%$ & 12 & na & $\begin{array}{l}\text { - PPI associated } \\
\text { with increased } \\
6 \text { y mortality } \\
\text { - Baseline RBBB } \\
\text { greater chance of } \\
\text { being dependent } \\
\text { at follow-up }\end{array}$ & na \\
\hline
\end{tabular}




\begin{tabular}{|c|c|c|c|c|c|c|c|c|c|}
\hline Study & $\begin{array}{l}\text { Indications } \\
\text { for PPI }\end{array}$ & $\begin{array}{c}\text { Timing } \\
\text { of PPI, } \mathbf{d}^{*}\end{array}$ & $\begin{array}{l}\text { PPI } \\
\text { rate }\end{array}$ & $\begin{array}{l}\text { Dependency } \\
\text { definition }\end{array}$ & $\begin{array}{l}\text { Dependency } \\
\text { rate }\end{array}$ & $\begin{array}{c}\text { Dependency } \\
\text { follow-up, } \\
\text { mo* }\end{array}$ & $\begin{array}{c}\text { Multivariable } \\
\text { predictors } \\
\text { of PPI }\end{array}$ & Association & $\begin{array}{c}\text { PPI-related } \\
\text { complications }\end{array}$ \\
\hline Dolci et $\mathrm{al}^{28}$ & $\begin{array}{l}80 \% \text { AVB } \\
11 \% \\
\text { Bradycardia } \\
9 \% \text { others }\end{array}$ & 4 & $13 \%$ & $\begin{array}{l}\text { "paced rhythm" } \\
\text { reported }\end{array}$ & $7 \%$ & 12 & $\begin{array}{l}\text { - Baseline RBBB } \\
\text { - QRS width } \\
\text { immediately } \\
\text { after TAVI }\end{array}$ & na & na \\
\hline 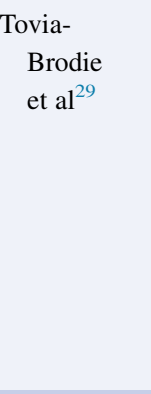 & $\begin{array}{l}92 \% \text { AVB } \\
8 \% \text { others }\end{array}$ & na & $8.8 \%$ & $\begin{array}{l}\text { No need for } \\
\text { ventricular pacing } \\
\text { defined as }<1 \% \\
\text { ventricular pacing } \\
\text { and intrinsic } 1: 1 \\
\text { AV conduction } \\
\text { with the device } \\
\text { programmed to } \\
\text { VVI } 30 \text { beats } \\
\text { per minute }\end{array}$ & $39 \%$ & 28,2 & $\begin{array}{l}\text { - Baseline long } \\
\text { PR interval } \\
\text { - Use of newer } \\
\text { generation } \\
\text { valves }\end{array}$ & na & $\begin{array}{l}3.7 \% \\
\quad \text { tamponade }\end{array}$ \\
\hline $\begin{array}{l}\text { Junquera } \\
\text { et }^{a^{30}}\end{array}$ & na & 6 & $12.7 \%$ & $\begin{array}{l}\mathrm{AVB} / \mathrm{CHB} \\
\text { recovery }= \\
\text { ventricular } \\
\text { pacing rate } \\
<1 \%\end{array}$ & $33.4 \%$ & 12 & na & na & na \\
\hline $\begin{array}{l}\text { Meduri } \\
\text { et }^{3 l^{31}}\end{array}$ & $\begin{array}{l}90 \% \text { AVB } \\
6 \% \\
\text { Bradycardia } \\
4 \% \text { others }\end{array}$ & 2 & $28.4 \%$ & $\begin{array}{l}\text { Patients who } \\
\text { were } \\
\text { symptomatic } \\
\text { or did not have } \\
\text { a native rhythm } \\
+ \\
\text { capture of the } \\
\text { percentage of } \\
\text { paced } \\
\text { ventricular } \\
\text { beats }\end{array}$ & $50 \%$ & 12 & $\begin{array}{l}\text { - Baseline RBBB } \\
\text { - Mean depth of } \\
\text { valve } \\
\text { implantation }\end{array}$ & $\begin{array}{l}\text { Medically treated } \\
\text { diabetes mellitus } \\
\text { in LOTUS valve } \\
\text { patients }\end{array}$ & na \\
\hline
\end{tabular}

\begin{tabular}{|c|c|c|c|c|c|c|c|c|c|}
\hline Total & $\begin{array}{l}82.7 \% \text { AVB } \\
2.7 \% \text { SSS } \\
2.8 \% \\
\text { bradycardia } \\
11.8 \% \text { Others }\end{array}$ & 3.2 & $17 \%$ & na & na & 11.8 & na & na & na \\
\hline
\end{tabular}




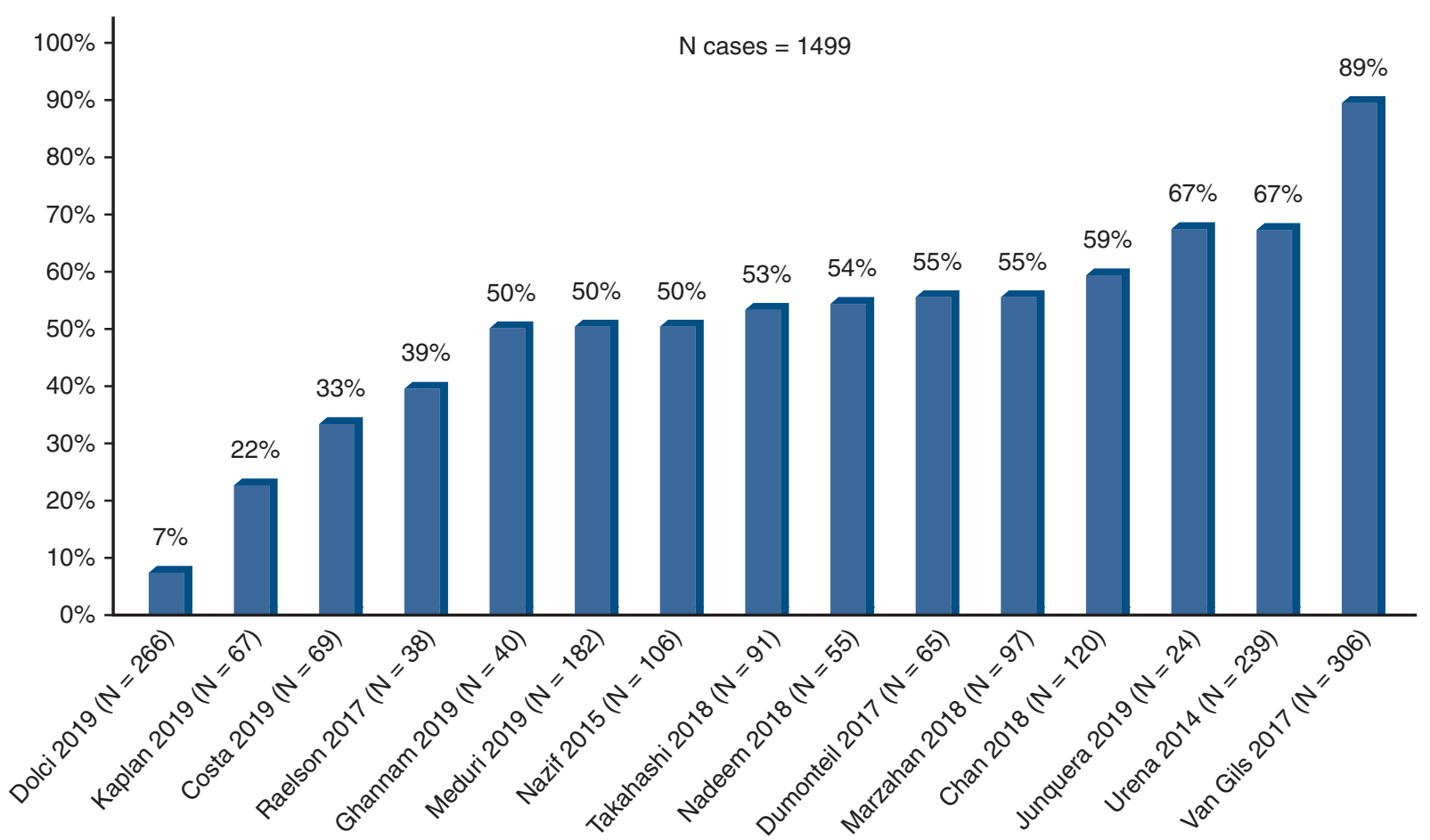

FIGURE 2. Pacemaker dependency at 1 year. Bars represent 1 -year pacemaker dependency. The rate of pacemaker dependency ranged from $7 \%$ to $89 \%$ in individual studies.

$80 \%-99 \%(48.3 \% ; 21.3 \%-76.5 \%)$, and $100 \%(53.8 \%$, $45.6 \%-61.7 \%), P=.427$.

\section{Complications and Multivariable Predictors of PPI}

Only 5 studies ${ }^{18,22,25,26,29}$ reported the PPI-related complications. The rate of PPI-related complications ranged from $1.2 \%{ }^{26}$ to $6 \% .^{25}$ The list of various complications is reported in Table 2. Information about predictors of PPI were provided by 7 studies (5319 patients). Pre-existing RBBB was the most frequent determinant factor of PPI. Regarding pacemaker dependency after TAVI at followup, multivariable analysis to investigate the predictors was performed in 5 studies: early PPI after TAVI, ${ }^{17}$ PPI on day $1,{ }^{27}$ and larger aortic annular size, ${ }^{26}$ were found as independent predictor of persistent atrioventricular conduction disturbances. In contrast, 2 studies failed to

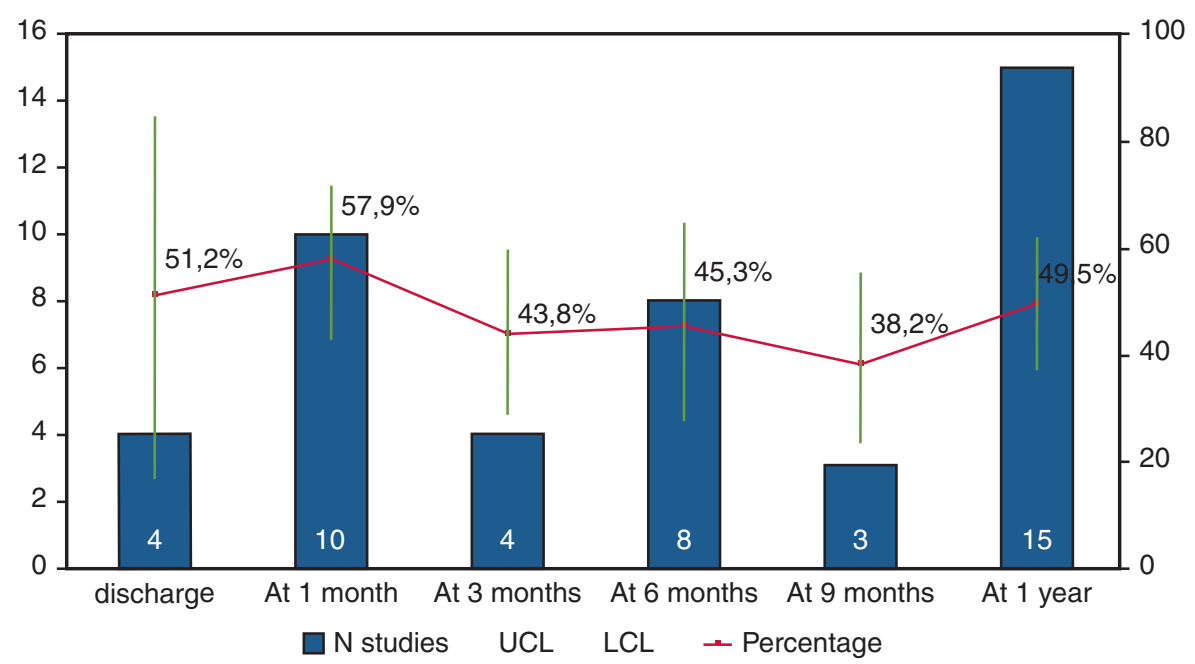

FIGURE 3. Rate of pacemaker dependency across the time after TAVI. Pooled percentage is reported with $95 \%$ confidence limits (blue line). Light blue bars represent number of studies. Yellow line is the interpolation line. UCL, Upper confidence limit; $L C L$, lower confidence limit. 


\begin{tabular}{|c|c|c|c|c|c|}
\hline Study & TE & SE & Weight & $\begin{array}{c}\text { Odds Ratio } \\
\text { IV, Random, } 95 \% \mathrm{CI}\end{array}$ & $\begin{array}{c}\text { Odds Ratio } \\
\text { IV, Random, } 95 \% \mathrm{CI}\end{array}$ \\
\hline Ghannam 2019 & -0.190 & 0.6200 & $15.0 \%$ & $0.827[0.245 ; 2.788]$ & \\
\hline Kaplan 2019 & -0.170 & 0.6700 & $13.1 \%$ & $0.844[0.227 ; 3.137]$ & \\
\hline Nadeem 2018 & 0.270 & 0.5400 & $18.9 \%$ & $1.310[0.455 ; 3.775]$ & \\
\hline Meduri 2019 & 1.240 & 0.9800 & $6.6 \%$ & $3.456[0.506 ; 23.589]$ & \\
\hline Chan 2018 & 1.550 & 0.5500 & $18.3 \%$ & $4.711[1.603 ; 13.846]$ & \\
\hline Costa 2019 & 0.890 & 0.6100 & $15.4 \%$ & $2.435[0.737 ; 8.049]$ & \\
\hline Raelson 2017 & 0.490 & 0.6800 & $12.8 \%$ & $1.632[0.431 ; 6.189]$ & \\
\hline Total $(95 \% \mathrm{Cl})$ & & & $100.0 \%$ & 1.760 [1.058; 2.927] & \\
\hline \multicolumn{5}{|c|}{ Heterogeneity: $\mathrm{Tau}^{2}=0.0653 ; \mathrm{Chi}^{2}=6.96, \mathrm{df}=6(P=.32) ; \mathrm{I}^{2}=14 \%$} & 0.10 .5 \\
\hline
\end{tabular}

FIGURE 4. Impact of baseline RBBB on 1-year rate of pacemaker dependency Forest plot. Patients with baseline RBBB have 2-fold greater risk to develop pacemaker dependency 1 year after TAVI. $T E$, Log odds ratio; $S E$, standard error; $I V$, weighted mean difference; $C I$, confidence limits; $P M$, pacemaker.

demonstrate significant predictors for atrioventricular conduction recovery or pacemaker dependency. ${ }^{13,22}$

\section{DISCUSSION}

The main findings of this systematic review regarding PPI and subsequent recovery of atrioventricular conduction or persistent pacemaker dependency in TAVI are as follows (Figure 7): (1) up to $50 \%$ of the patients with PPI after TAVI exhibit no pacemaker dependency at 1 year follow-up; (2) patients with baseline RBBB and (3) SE prosthesis have 2-fold greater risk to develop pacemaker dependency 1 year after TAVI; and (4) baseline AF does not influence the risk of pacemaker dependency at 1 year.

Several recent reports show that atrioventricular conduction defects requiring PPI post-TAVI may involve as much as $30 \%$ of the treated patients, therefore representing the most frequent complication in such a setting. ${ }^{16}$ However, nearly $50 \%$ of such patients are not pacemaker-dependent at 1-year follow-up, with recovery of atrioventricular conduction occurring even at a very early stage after implant, like before hospital discharge. ${ }^{28}$ Nonetheless, the range of postimplant pacemaker dependency or effective atrioventricular conduction restoration varies largely, ranging from $7 \%{ }^{28}$ to $89 \% .^{12}$ This wide interval could be explained by heterogeneity and lack of consensus on pacemaker dependency definition. This limitation negatively influenced the study data interpretation in our analysis. Furthermore, pacemaker dependency may intermittently occur, thereby characterizing a different pattern of pacing dependency. This peculiar aspect was not available but might represent an additional factor to be investigated.

In our analysis, patients receiving SE valves and presenting with a preoperative RBBB had a 2 -fold greater risk to have persistent pacing dependency at 1 year after TAVI. Ramazzina and colleagues (Table E2) showed that atrioventricular block as indication for PPI was always associated with pacemaker dependency at follow-up. In contrast, Gaede and colleagues ${ }^{17}$ demonstrated a low rate of longterm persistence of atrioventricular block after TAVI procedure. A few studies address investigate predictors of pacemaker dependency after TAVI. Naveh and colleagues (Table E2) showed that baseline RBBB, long post-TAVI PR interval, and delayed PR interval from baseline were independent predictors for long-term pacemaker dependency. Sharma and colleagues (Table E2) showed that bifascicular block, RBBB, intraprocedural complete heart block, and QRS duration $>120$ milliseconds were associated with pacemaker dependency at 30-day follow-up. The impact

\begin{tabular}{|c|c|c|c|c|c|}
\hline Study & TE & SE & Weight & $\begin{array}{c}\text { Odds Ratio } \\
\text { IV, Random, } 95 \% \text { Cl }\end{array}$ & $\begin{array}{c}\text { Odds Ratio } \\
\text { IV, Random, } 95 \% \mathrm{CI}\end{array}$ \\
\hline Chan 2018 & -0.920 & 1.1700 & $9.9 \%$ & $0.399[0.040 ; 3.948]$ & \\
\hline Kaplan 2019 & -0.120 & 0.9800 & $14.1 \%$ & $0.887[0.130 ; 6.054]$ & \\
\hline Nadeem 2018 & 0.570 & 0.9100 & $16.4 \%$ & $1.768[0.297 ; 10.523]$ & \\
\hline Raelson 2017 & 0.570 & 0.6700 & $30.2 \%$ & $1.768[0.476 ; 6.574]$ & \\
\hline Ghannam 2019 & 1.300 & 0.6800 & $29.3 \%$ & $3.669[0.968 ; 13.912]$ & \\
\hline Total $(95 \% \mathrm{Cl})$ & & & $100.0 \%$ & $1.714[0.833 ; 3.529]$ & \\
\hline \multirow{2}{*}{\multicolumn{5}{|c|}{ Heterogeneity: $\mathrm{Tau}^{2}=0 ; \mathrm{Chi}^{2}=3.26, \mathrm{df}=4(P=.51) ;\left.\right|^{2}=0 \%$}} & 0.10 .512 \\
\hline & & & & & 1-y PM depen \\
\hline
\end{tabular}

FIGURE 5. Impact of baseline AF on 1-year rate of pacemaker dependency. Forest plot. Patients with baseline AF have no greater risk to develop pacemaker dependency 1 year after TAVI. TE, Log odds ratio; $S E$, standard error; $I V$, weighted mean difference; $C I$, confidence limits; $P M$, pacemaker. 


\begin{tabular}{|c|c|c|c|c|c|c|c|}
\hline Study & TE & SE & Weight & $\begin{array}{c}\text { Odds Ratio } \\
\text { IV, Random, } 95 \% \text { CI }\end{array}$ & $\begin{array}{r}\text { Odd } \\
\text { IV, Rand }\end{array}$ & $\begin{array}{l}\text { Is Ratio } \\
\text { lom, } 95 \% \mathrm{Cl}\end{array}$ & \\
\hline Raelson 2017 & -0.190 & 0.6700 & $20.2 \%$ & $0.827[0.222 ; 3.075]$ & & & \\
\hline Nadeem 2018 & 0.260 & 0.5900 & $23.7 \%$ & $1.297[0.408 ; 4.122]$ & & & \\
\hline Urena 2014 & 1.160 & 0.3100 & $41.9 \%$ & $3.190[1.737 ; 5.857]$ & & & \\
\hline Kaplan 2019 & 1.630 & 0.8600 & $14.2 \%$ & $5.104[0.946 ; 27.538]$ & & & \\
\hline Total $(95 \% \mathrm{Cl})$ & & & $100.0 \%$ & $2.096[1.012 ; 4.341]$ & & & \\
\hline \multicolumn{5}{|c|}{ Heterogeneity: $\mathrm{Tau}^{2}=0.2334 ; \mathrm{Chi}^{2}=5.23, \mathrm{df}=3(P=.16) ; \mathrm{I}^{2}=43 \%$} & 0.1 & 12 & 10 \\
\hline
\end{tabular}

FIGURE 6. Impact of SE prosthesis (vs BE) on 1-year rate of pacemaker dependency. Forest plot. Patients with SE prosthesis have 2-fold greater risk to develop pacemaker dependency at 1 year after TAVI. TE, Log odds ratio; $S E$, standard error; $I V$, weighted mean difference; $C I$, confidence limits; $P M$, pacemaker.

of baseline AF on pacemaker dependency at follow-up is controversial. Early PPI after TAVI procedure was the strongest predictor regarding persistent atrioventricular block and pacemaker dependency, based on large sample-size studies. ${ }^{17,27}$ According to these findings, we should emphasize that patients without baseline characteristics potentially leading to pacemaker dependency should benefit from other temporary leadless system as Micra AV (Medtronic, Minneapolis, Minn) to reduce the rate of permanent pacemaker implanted, as the Micra AV system is recently found to be safe; efficient and as performant as transvenous single-chamber pacemaker. ${ }^{32,33}$

Nowadays, guidelines regarding timing of PPI after TAVI are rather cloudy and not based on thorough clinical investigations. ${ }^{8,34}$ Due to the lack of consistent data, the dilemma about the appropriate timing for pacemaker implantation after TAVI is left to the discretion of the attending cardiologist according to the different centers' policies and therefore is associated with extreme variability in clinical management. Erkapic and colleagues ${ }^{35}$ showed that atrioventricular block occurs in more than $90 \%$ of the cases within the first postTAVI week, which would allow to monitor carefully the patients at least seven days before considering PPI. However, some others studies support early PPI after TAVI procedure. ${ }^{30}$ Actually, as the optimal timing for PPI after TAVI is not established, the variability of decision certainly influences the outcome of pacemaker dependency, making difficult to conclude about the best interval to proceed to a definitive PPI.

PPI after TAVI is associated with increased long-term mortality. ${ }^{27}$ Faroux and colleagues ${ }^{5}$ show the negative impact of PPI after TAVI on survival and heart failure hospitalization within the year following TAVI. Xi and colleagues $^{7}$ also show a greater all-cause mortality in TAVI patients receiving a PPI. In this systematic review, only 4 studies addressed the PPI-related complications and only 8 studies reported the 30-day mortality, making the overall appraisal of the impact of PPI on patient outcome likely underestimated, as emphasized by the Danish experience of Kirkfeldt and colleagues. ${ }^{36}$ Report on PPI-related complications is also markedly variable ${ }^{37}$ and the lack of a standardize international classification of these complications may further contribute to PPI-related complications and related outcome actually under-reported.

\section{Limitations}

This systematic review had several limitations that should be acknowledged. First, the follow-up varied remarkably among the included studies. The 1-year dependency was not available for all studies, limiting the conclusion to only 15 studies, whereas the others publications provided a follow-up mainly to 1 to 3 months. Only 3 studies presented a longer follow-up up to 4 years. Second, the heterogeneity about pacemaker dependency definition in the studies semantically limits our ability to compare the studies. Third, although several studies identified some risk-factors for PPI and only 1 study states multivariable predictors of pacemaker dependency, there was no agreement between studies. Finally, this is a systematic review of the literature; analysis of individual patients-data level may provide further understandings: in many of studies it was not specifically clear if the patient's native rhythm was assessed and thereby knowing for certain which patients truly required PPI.

\section{CONCLUSIONS}

This systematic review investigates the rate and predictors of pacing dependency after TAVI. Data from literature show that almost one half of the pacemakers are actively operating at 1-year follow-up. Baseline RBBB and SE valves are associated with a greater rate of pacemaker dependency at follow-up. These findings suggest that atrioventricular conduction disturbances after TAVI are reversible in a large percentage of patients. Such a condition may occur at variable time after the TAVI procedure, even within the TAVI-related hospitalization and, therefore, at a very early stage. These findings clearly indicate the need of thorough investigations regarding timing of pacemaker implantation, recovery of atrioventricular 


\section{One Year Pacing Dependency After Pacemaker Implantation in TAVI Patients: Systematic Review and Meta-Analysis}

23 studies analyzed, involving

18,610 patients undergoing

TAVI

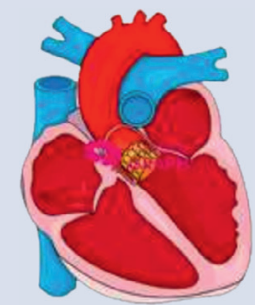

Crude incidence of conduction disturbances leading to PPI of $17 \%(8.8 \%$ to $32 \%)$

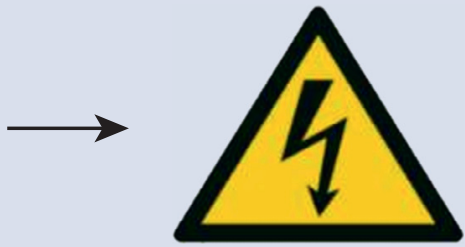

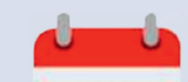

1 $50 \%$ of the patients free from pacemaker dependency at 1 year

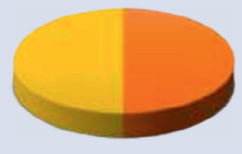

PUBMED, EMBASE, MEDLINE

Up to $50 \%$ of the patients with permanent pacemaker implantation following TAVI exhibits no pacemaker dependency at 1-year follow-up.

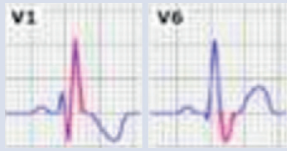

Patients with baseline RBBB

have 2-fold higher risk to develop pacemaker dependency 1 year after TAVI

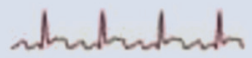

Patients with baseline AF have no higher risk to develop pacemaker dependency 1 year after TAVI

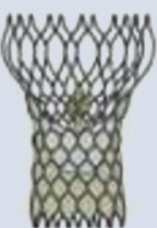

Patients with SE prosthesis have 2 -fold higher risk to develop pacemaker dependency at 1 year after TAVI

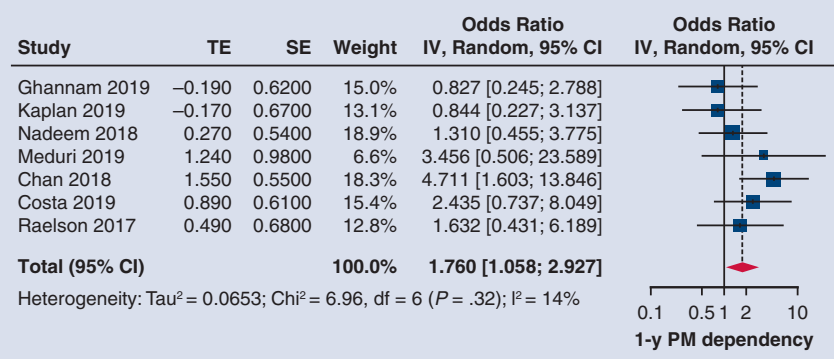

\begin{tabular}{|c|c|c|c|c|c|}
\hline Study & TE & SE & Weight & $\begin{array}{c}\text { Odds Ratio } \\
\text { IV, Random, } 95 \% \mathrm{Cl}\end{array}$ & $\begin{array}{c}\text { Odds Ratio } \\
\text { IV, Random, } 95 \% \mathrm{CI}\end{array}$ \\
\hline Chan 2018 & -0.920 & 1.1700 & $9.9 \%$ & $0.399[0.040 ; 3.948]$ & \\
\hline Kaplan 2019 & -0.120 & 0.9800 & $14.1 \%$ & $0.887[0.130 ; 6.054]$ & \\
\hline Nadeem 2018 & 0.570 & 0.9100 & $16.4 \%$ & $1.768[0.297 ; 10.523]$ & \\
\hline Raelson 2017 & 0.570 & 0.6700 & $30.2 \%$ & $1.768[0.476 ; 6.574]$ & \\
\hline Ghannam 2019 & 1.300 & 0.6800 & $29.3 \%$ & $3.669[0.968 ; 13.912]$ & \\
\hline Total $(95 \% \mathrm{Cl})$ & & & $100.0 \%$ & 1.714 [0.833; 3.529] & \\
\hline \multicolumn{5}{|c|}{ Heterogeneity: $\mathrm{Tau}^{2}=0 ; \mathrm{Chi}^{2}=3.26, \mathrm{df}=4(P=.51) ; 1^{2}=0 \%$} & $0.1 \quad 0.512$ \\
\hline
\end{tabular}

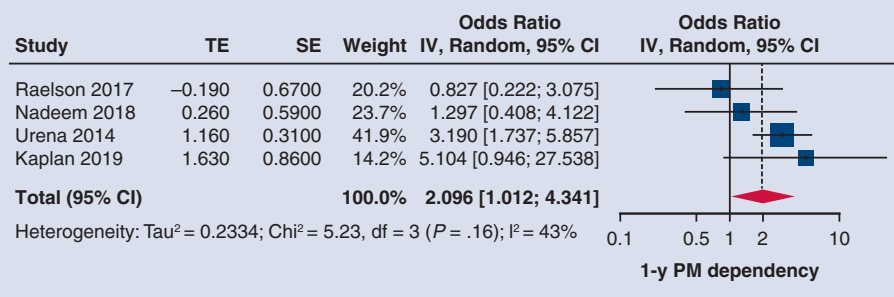

FIGURE 7. Up to $50 \%$ of the patients with permanent pacemaker implantation following TAVI exhibits no pacemaker dependency at 1-year follow-up. $T A V I$, Transcatheter aortic valve implantation; $P P I$, permanent pacemaker implantation; RBBB, right bundle branch block; $T E$, log odds ratio; $S E$, standard error; $I V$, weighted mean difference; $C I$, confidence limits; $P M$, pacemaker.

conduction, and predictors of pacemaker dependence as endpoints for further studies.

\section{Conflict of Interest Statement}

Dr Vernooy reported research grant from Medtronic. Dr Van't Hof reported grants from Medtronic, Astra Zeneca, Boehringer Ingelheim, and Abbott. Dr Lorusso reported grants from Medtronic, LivaNova, and Eurosets. All other authors reported no conflicts of interest.

The Journal policy requires editors and reviewers to disclose conflicts of interest and to decline handling or reviewing manuscripts for which they may have a conflict of interest. The editors and reviewers of this article have no conflicts of interest. 


\section{References}

1. Cribier A, Eltchaninoff H, Bash A, Borenstein N, Tron N, Bauer F, et al. Percutaneous transcatheter implantation of an aortic valve prosthesis for calcific aortic stenosis: first human case description. Circulation. 2002;106:3006-8.

2. Leon MB, Smith CR, Mack M, Miller C, Moses JW, Svensson LG, et al. Transcatheter aortic-valve implantation for aortic stenosis in patients who cannot undergo surgery. N Engl J Med. 2010;363:1597-607.

3. Kheiri B, Osman M, Abubakar H, Subahi A, Chachine A, Ahmed S, et al. Transcatheter versus surgical aortic valve replacement in low-risk surgical patients: a meta-analysis of randomized clinical trials. Cardiovasc Revasc Med. 2019;20: 838-42.

4. Van Rosendael PJ, Delgado V, Bax JJ. Pacemaker implantation rate after transcatheter aortic valve implantation with early and new-generation devices: a systematic review. Eur Heart J. 2018;39:2003-13.

5. Faroux L, Chen S, Muntané-Carol G, Reguerio A, Philippon F, Sondergaard L, et al. Clinical impact of conduction disturbances in transcatheter aortic valve replacement recipients: a systematic review and meta-analysis. Eur Heart J. 2020;41:2771-81.

6. Piazza N, Nuis R-J, Tzikas A, Otten A, Onuma Y, Garia-Garcia H, et al. Persistent conduction abnormalities and requirements for pacemaking six months after transcatheter aortic valve implantation. Eurointervention. 2010;6:475-84.

7. Xi Z, Liu T, Liang J, Zhou UJ, Liu W. Impact on postprocedural permanent pacemaker implantation on clinical outcomes after transcatheter aortic valve replacement: a systematic review and meta-analysis. J Thorac Dis. 2019;11:5130-9.

8. Kusumoto FM, Schoenfeld MH, Barrett C, Edgerton JR, Ellenbogen KA, Gold MR, et al. 2018 ACC/AHA/HRS guideline on the evaluation and management of patients with bradycardia and cardiac conduction delay: a report of the American College of Cardiology/American Heart Association task force on clinical practice guidelines and the Heart Rhythm Society. Circulation. 2019;140:e382-482.

9. Bjerre Thygesen J, Loh PH, Cholteesupachai J, Franzen O, Sondergaard L. Reevaluation of the indications for permanent pacemaker implantation after transcatheter aortic valve implantation. J Invasive Cardiol. 2014;26:94-8.

10. Urena M, Webb JG, Tamburino C, Munoz-Garcia AJ, Cheema A, Dager AE, et al. Permanent pacemaker implantation after transcatheter aortic valve implantation: impact on late clinical outcomes and left ventricular function. Circulation. 2014;129:1233-43.

11. Nazif TM, Dizon JM, Hahn RT, Xu K, Babaliaros V, Douglas PS, et al. Predictors and clinical outcomes of permanent pacemaker implantation after transcatheter aortic valve replacement: the PARTNER (Placement of AoRtic TraNscathertER Valves) trial and registry. JACC Cardiovasc Interv. 2015;8:60-9.

12. Van Gils L, Tchetche D, Lhermusier T, Abawi M, Dumonteuil N, RodriguezOlivares R, et al. Transcatheter heart valve selection and permanent pacemaker implantation in patients with pre-existent right bundle branch block. J Am Heart Assoc. 2017;6:e05028.

13. Raelson CA, Gabriels J, Ruan J, Ip JE, Thomas G, Liu CF, et al. Recovery of atrioventricular conduction in patients with heart block after transcatheter aortic valve replacement. J Cardiovasc Electrophysiol. 2017;28:1196-202.

14. Dumonteil N, Meredith IT, Blackman DJ, Tchétché D, Hildick-Smith D, Spence MS, et al. Insights into the need for permanent pacemaker following implantation of the repositionable LOTUS valve for transcatheter aortic valve replacement in 250 patients: results from the REPRISE II trial with extended cohort. Eurointervention. 2017;13:796-803.

15. Kaplan RM, Yadlapati A, Cantey EP, Passman RS, Gajjar M, Knight BP, et al. Conduction recovery following pacemaker implantation after transcatheter aortic valve replacement. Pacing Clin Electrophysiol. 2019;42:146-52.

16. Chamandi C, Barbanti M, Munoz-Garcia A, Latib A, Nombela-Franco L, Guttiérrez-Ibanez E, et al. Long-term outcomes in patients with new permanent pacemaker implantation following transcatheter aortic valve replacement. JACC Cardiovasc Interv. 2018;11:301-10.

17. Gaede L, Kim WK, Liebetrau C, Dörr O, Sperzel J, Blumenstein J, et al. Pacemaker implantation after TAVI: predictors of AV block persistence. Clin Res Cardiol. 2018;107:60-9.

18. Gonska B, Keßler M, Wöhrle J, Rottbauer W, Seeger J. Influence of permanent pacemaker implantation after transcatheter aortic valve implantation with newgeneration devices. Neth Heart J. 2018;26:620-7.

19. Marzahn C, Koban C, Seifert M, Isotani A, Neub M, Hölschermann F, et al. Conduction recovery and avoidance of permanent pacing after transcatheter aortic valve implantation. J Cardiol. 2018;71:101-8.

20. Nadeem F, Tsushima T, Ladas TP, Thomas RB, Patel SM, Saric P, et al. Impact of right ventricular pacing in patients who underwent implantation of permanent pacemaker after transcatheter aortic valve implantation. Am J Cardiol. 2018 122:1712-7.

21. Campelo-Parada F, Nombela-Franco L, Urena M, Regueiro A, JiménezQuevedo P, Del Trigo M, et al. Timing of onset and outcome of new conduction abnormalities following transcatheter aortic valve implantation: role of balloon aortic valvuloplasty. Rev Esp Cardiol (Eng Ed). 2018;71:162-9.

22. Mirolo A, Viart G, Durand E, Savouré A, Godin B, Auquier N, et al. Pacemaker memory in post-TAVI patients: who should benefit from permanent pacemaker implantation? Pacing Clin Electrophysiol. 2018;41:1178-84.

23. Van Gils L, Baart S, Kroon H, Rahhab Z, El Faquir N, Rodriguez-Olivares R, et al. Conduction dynamics after transcatheter aortic valve implantation and implications for permanent pacemaker implantation and early discharge: the CONDUCT-study. Europace. 2018;20:1981-8.

24. Takahashi M, Badenco N, Monteau J, Gandjbakhch E, Extramiana F, Urena M et al. Impact of pacemaker mode in patients with atrioventricular conduction disturbance after trans-catheter aortic valve implantation. Catheter Cardiovasc Interv. 2018;92:1380-6.

25. Chan WK, Danon A, Wijeysundera HC, Singh SM. Single versus dual lead atrioventricular sequential pacing for acquired atrioventricular block during transcatheter aortic valve implantation procedures. Am J Cardiol. 2018;122:633-7.

26. Ghannam M, Cunnane R, Menees D, Grossman MP, Chetcuti S, Patel H, et al Atrioventricular conduction in patients undergoing pacemaker implant following self-expandable transcatheter aortic valve replacement. Pacing Clin Electrophysiol. 2019;42:980-8

27. Costa G, Zappulla P, Barbanti M, Cirasa A, Todaro D, Rapisarda G, et al. Pacemaker dependency after transcatheter aortic valve implantation: incidence, predictors and long-term outcomes. Eurointervention. 2019;15:875-83.

28. Dolci G, Vollema EM, Van Der Kley F, De Weger A, Ajmone Marsan N, Delgado $\mathrm{V}$, et al. One-year follow-up of conduction abnormalities after transcatheter aortic valve implantation with the SAPIEN 3 valve. Am J Cardiol. 2019;124 $1239-45$.

29. Tovia-Brodie O, Letourneau-Shesaf S, Hochstadt A, Steinvil A, Rosso R Finkelstein A, et al. The utility of prophylactic pacemaker implantation in right bundle branch block patient pre-transcatheter aortic valve implantation. Isr Med Assoc J. 2019;12:790-5.

30. Junquera L, Freitas-Ferraz AB, Padron R, Silva I, Nunes-Ferreira-Neto A, Guimaraes L, et al. Intraprocedural high-degree atrioventricular block or complete heart block in transcatheter aortic valve replacement recipients with no prior intraventricular conduction disturbance. Catheter Cardiovasc Interv. 2020;95:982-90.

31. Meduri CU, Kereiakes DJ, Rajagopal V, Makkar RR, O’Hair D, Linke A, et al Pacemaker implantation and dependency after transcatheter aortic valve replacement in the REPRISE III Trial. J Am Heart Assoc. 2019;8:e012594.

32. Moore SKL, Chau KH, Chaudhary S, Rubin G, Bayne J, Avula UMR, et al. Leadless pacemaker implantation: a feasible and reasonable option in transcatheter heart valve replacement patients. Pacing Clin Electrophysiol. 2019;42:542-7.

33. Garweg C, Vandenberk B, Foulon S, Poels P, Haemers P, Ector J, et al. Leadless pacemaker for patients following cardiac valve intervention. Arch Cardiovasc Dis. 2020;113:772-9.

34. Brignole M, Auricchio A, Baron-Esquivias G, Bordachar P, Boriani G, Breithardt O-A, et al. 2013 ESC guidelines on cardiac pacing and cardiac resynchronization therapy: the task force on cardiac pacing and resynchronization therapy of the European Society of Cardiology (ESC). Developed in Collaboration with the European Heart Rhythm Association (EHRA). Europace. 2013;15: 1070-118.

35. Erkapic D, De Rosa S, Kelava A, Lehmann R, Fichtlscherer S, Hohnloser SH. Risk for permanent pacemaker after transcatheter aortic valve implantation: a comprehensive analysis of the literature. J Cardiovasc Electrophysiol. 2012; 23:391-7.

36. Kirkfeldt RE, Johansen JB, Nohr EA, Jorgensen OD, Nielsen JC. Complications after cardiac implantable electronic device implantations: an analysis of a complete, nationwide cohort in Denmark. Eur Heart J. 2014:35:1186-94.

37. Ezzat VA, Lee V, Ahsan SN, Chow AW, Segal O, Rowland E, et al. A systematic review of ICD complications in randomized controlled trials versus registries: is our 'real-world' data an underestimation? Open Heart. 2015;2:e000198.

Key Words: conduction disturbances, pacemaker dependency, permanent pacemaker, transcatheter aortic valve implantation 


\section{References of low-sample studies including $<250$ patients}

E1. Sinhal A, Altwegg L, Pasupati S, Humphries KH, Allard M, Martin P, et al. Atrioventricular block after transcatheter balloon expandable aortic valve implantation. JACC Cardiovasc Interv. 2008;1:305-9.

E2. Jilaihawi H, Chin D, Vasa-Nicotera M, Jeilan M, Spyt T, Ng GA, et al. Predictors for permanent pacemaker requirement after transcatheter aortic valve implantation with the CoreValve bioprosthesis. Am Heart J. 2009;157: 860-6.

E3. Baan J Jr, Yong ZY, Koch KT, Henriques JP, Bouma BJ, Vis MM, et al. Factors associated with cardiac conduction disorders and permanent pacemaker implantation after percutaneous aortic valve implantation with the CoreValve prosthesis. Am Heart J. 2010;159:497-503.

E4. Fraccaro C, Buja G, Tarantini G, Gasparetto V, Leoni L, Razzolini R, et al. Incidence, predictors and outcomes of conduction disorders after transcatheter self-expandable aortic valve implantation. Am J Cardiol. 2011; 107:747-54.

E5. Van der Boon RMA, Van Mieghem NM, Theuns DA, Nuis RJ, Nauta ST, Srruys PW, et al. Pacemaker dependency after trans-catheter aortic valve implantation with the self-expanding Medtronic CoreValve System. Int J Cardiol. 2013;168:1269-73.

E6. Pereira E, Ferreira N, Caeiro D, Primo J, Adao L, Oliveira L, et al. Transcatheter aortic valve implantation and requirements of pacing over time. Pacing Clin Electrophysiol. 2013;36:559-69.

E7. Goldenberg G, Kusniec J, Kadmon E, Golovchiner G, Zabarsky R, Nevzorov R, et al. Pacemaker implantation after transcatheter aortic valve implantation. Am J Cardiol. 2013;112:1632-4.

E8. Ramazzina C, Knecht S, Jeger R, Kaiser C, Schaer B, Osswald S, et al. Pacemaker implantation and need for ventricular pacing during follow-up after transcatheter aortic valve implantation. Pacing Clin Electrophysiol. 2014;37: $1592-601$.

E9. Boerlage-Van Dijk L, Kooiman KM, Yong ZY, Wiegerinck EM, Damman P, Bouma BJ, et al. Predictors and permanency of cardiac conduction disorders and necessity of pacing after transcatheter aortic valve implantation. Pacing Clin Electrophysiol. 2014;37:1520-9.

E10. Renilla A, Rubin JM, Rozado J, Moris C. Long-term evolution of pacemaker dependency after percutaneous aortic valve implantation with the CoreValve prosthesis. Int J Cardiol. 2015;201:61-3.

E11. Petronio AS, Sinning JM, Van Mieghem N, Zucchelli G, Nickenig G, Bekeredjian R, et al. Optimal implantation depth and adherence to guidelines on permanent pacing to improve the results of transcatheter aortic valve replacement with the Medtronic CoreValve System: the CoreValve Prospective, International, Post-Market ADVANCE-II study. JACC Cardiovasc Interv. 2015;8: 837-46.

E12. Weber M, Brüggemann E, Schueler R, Momcilovic D, Sinning JM, Ghanem A, et al. Impact of left ventricular conduction defect with or without need for permanent right ventricular pacing on functional and clinical recovery after TAVR. Clin Res Cardiol. 2015;104:964-74.

E13. Schernthaner C, Kraus J, Danmayr F, Hammerer M, Schneider J, Hoppe UC, et al. Short-term pacemaker dependency after transcatheter aortic valve implantation. Wien Klin Wochenschr. 2016;128:198-203.

E14. Kostopoulou A, Karyofillis P, Livanis E, Thomopoulou S, Stefopoulos C, Doudoumis K, et al. Permanent pacing after transcatheter aortic valve implantation of a CoreValve prosthesis as determined by electrocardiographic and electrophysiological predictors: a single-centre experience. Europace. 2016; 18:131-7.
E15. Sideris S, Benetos G, Toutouzas K, Drakopoulou M, Sotiropoulos E, Gatzoulis K, et al. Outcomes of same day pacemaker implantation after TAVI. Pacing Clin Electrophysiol. 2016;39:690-5.

E16. Luke D, Huntsinger M, Carlson SK, Lin R, Tun H, Matthews RV, et al. Incidence and predictors of pacemaker implant post commercial approval of the CoreValve system for TAVR. J Innov Card Rhythm Manag. 2016;7:2452-60.

E17. Makki N, Dollery J, Jones D, Crestanello J, Lilly S. Conduction distrubances after TAVR: electrophysiological studies and pacemaker dependency. Cardiovasc Revasc Med. 2017;18:S10-3.

E18. Nijenhuis VJ, Van Dijk VF, Chaldoupi SM, Balt JC, Ten Berg JM. Severe conduction defects requiring permanent pacemaker implantation in patients with a new-onset left bundle branch block after transcatheter aortic valve implantation. Europace. 2017;19:1015-21.

E19. Naveh S, Perlman GY, Elitsur Y, Planer D, Gilon D, Leibowitz D, et al. Electrocardiographic predictors of long-term cardiac pacing dependency following transcatheter aortic valve implantation. J Cardiovasc Electrophysiol. 2017;28:216-23.

E20. Alasti M, Rashid H, Rangasamy K, Kotschet E, Adam D, Alison J, et al. Longterm pacemaker dependency and impact of pacing on mortality following transcatheter aortic valve replacement with the LOTUS valve. Catheter Cardiovasc Interv. 2018;92:777-82.

E21. Ortak J, D'Ancona G, Ince H, Agma HU, Sarak E, Öner A, et al. Transcatheter aortic valve implantation with a mechanically expandable prosthesis: a learning experience for permanent pacemaker implantation rate reduction. Eur J Med Res. 2018;23:14.

E22. Rodes-Cabau J, Urena M, Nombela-Franco L, Amat-Santos I, Kleiman N, Munoz-Garcia AJ, et al. Arrhythmic burden as determined by ambulatory continuous cardiac monitoring in patients with new-onset persistent left bundle branch block following transcatheter aortic valve replacement: the MARE study. JACC Cardiovasc Interv. 2018;11:1495-505.

E23. Leong D, Sovari AA, Ehdaie A, Chakravarty T, Liu Q, Jilaihawi H, et al. Permanent-temporary pacemakers in the management of patients with conduction abnormalities after transcatheter aortic valve replacement. J Interv Card Electrophysiol. 2018;52:111-6.

E24. Sharma E, Chu AF. Predictors of right ventricular pacing and pacemaker dependence in transcatheter aortic valve replacement patients. J Interv Card Electrophysiol. 2018;51:77-86.

E25. Bacik P, Poliacikova P, Kaliska G. Who needs a permanent pacemaker after transcatheter aortic valve implantation? Bratisl Lek Listy. 2018;119:560-5.

E26. Megaly M, Gössl M, Sorajja P, Anzia LE, Henstrom J, Morley P, et al. Outcomes after pacemaker implantation in patients with new-onset left bundlebranch block after transcatheter aortic valve replacement. Am Heart J. 2019; 218:128-32.

E27. Yazdchi F, Hirji S, Kaneko T. Quality control for permanent pacemaker implantation after transcatheter aortic valve replacement. Ann Thorac Surg. 2020;110: 347-8.

E28. McCaffrey JA, Alzahrani T, Datta T, Solomon AJ, Mercader M, Mazhari R, et al. Outcomes of acute conduction abnormalities following transcatheter aortic valve implantation with a balloon expandable valve and predictors of delayed conduction system abnormalities in follow-up. Am J Cardiol. 2019;123:1845-52.

E29. Miura M, Shirai S, Uemura T, Hayashi M, Takiguchi H, Ito S, et al. Clinical impact of intraventricular conduction abnormalities after transcatheter aortic valve implantation with balloon-expandable valves. Am J Cardiol. 2019;123: 297-305.

E30. Dhakal BP, Skinner KA, Kumar K, Lotun K, Shetty R, Kazui T, et al. Arrhythmias in relation to mortality after transcatheter aortic valve replacement. Am J Med. 2020;133:1336-42.e1. 


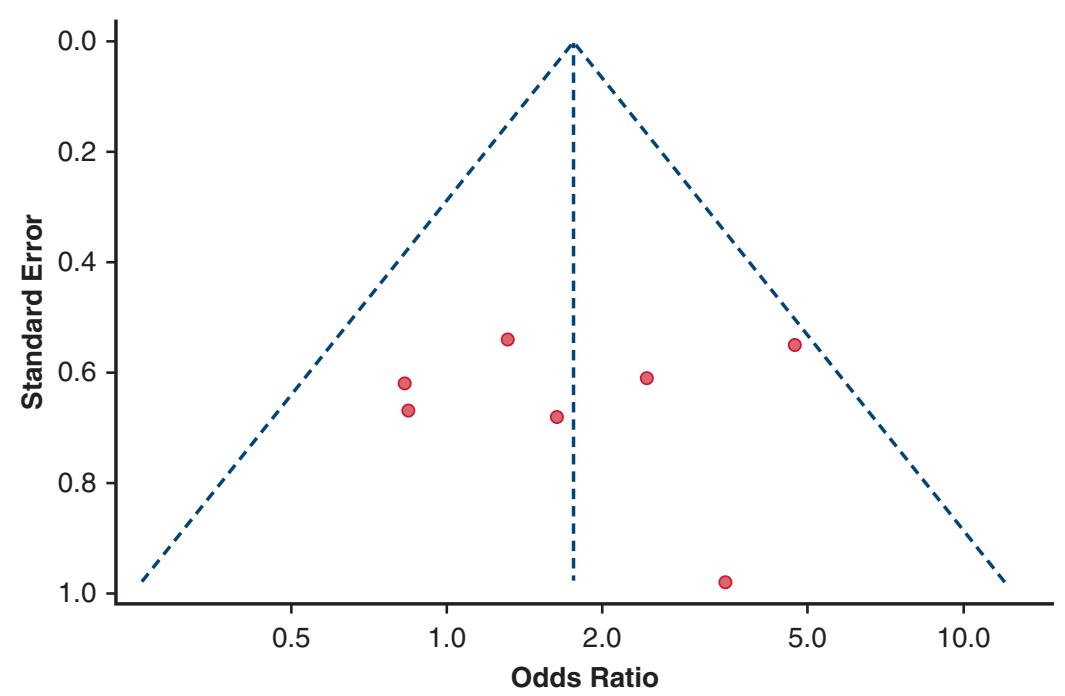

FIGURE E1. Funnel plot on the impact of baseline RBBB on 1-year rate of pacemaker dependency. No publication bias was found among studies reporting the influence of baseline RBBB on pacemaker dependency.

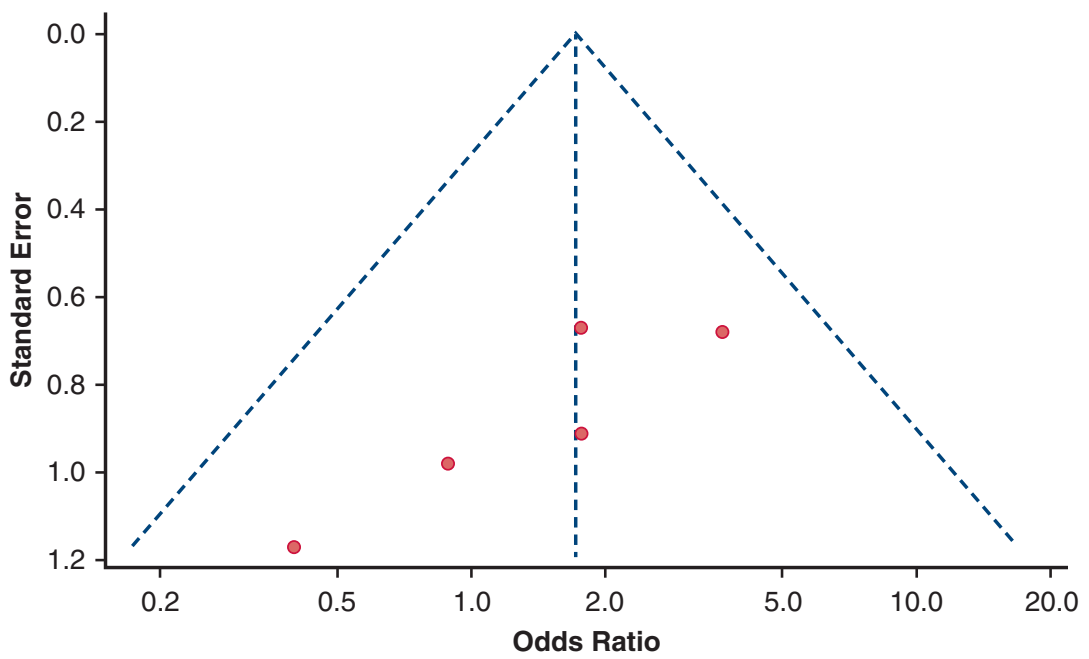

FIGURE E2. Funnel plot on the influence of baseline AF on pacemaker dependency. No publication bias was found among studies reporting the influence of baseline AF on pacemaker dependency. 


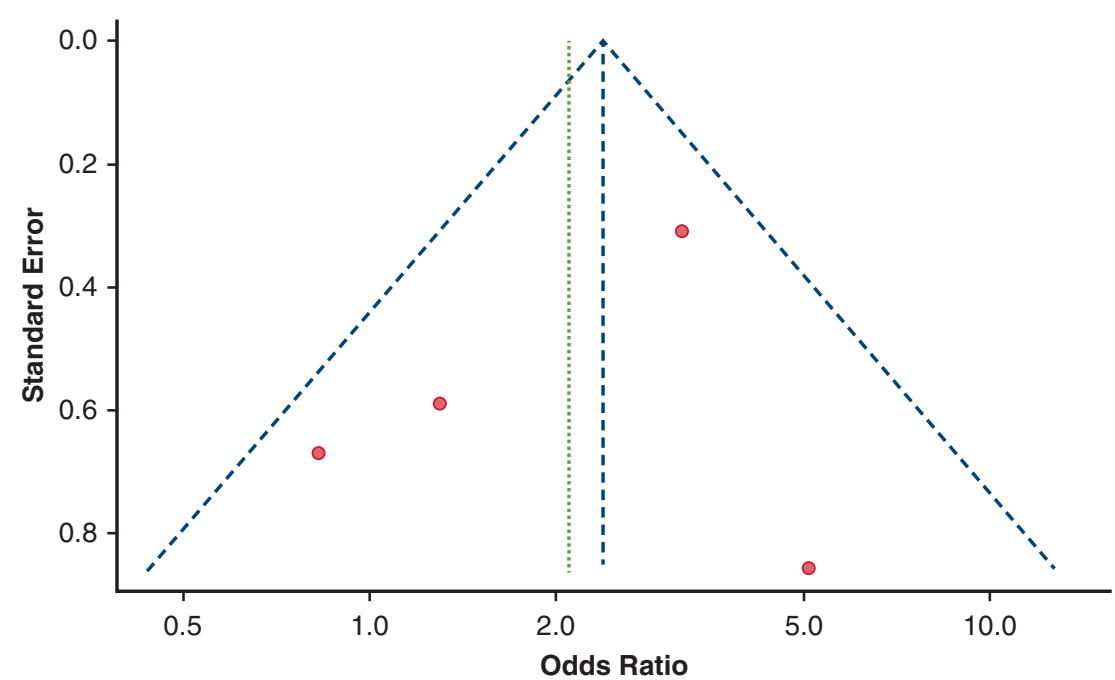

FIGURE E3. Funnel plot on the influence of SE versus BE valves on pacemaker dependency. No publication bias was found among studies reporting the influence of SE versus BE on pacemaker dependency.

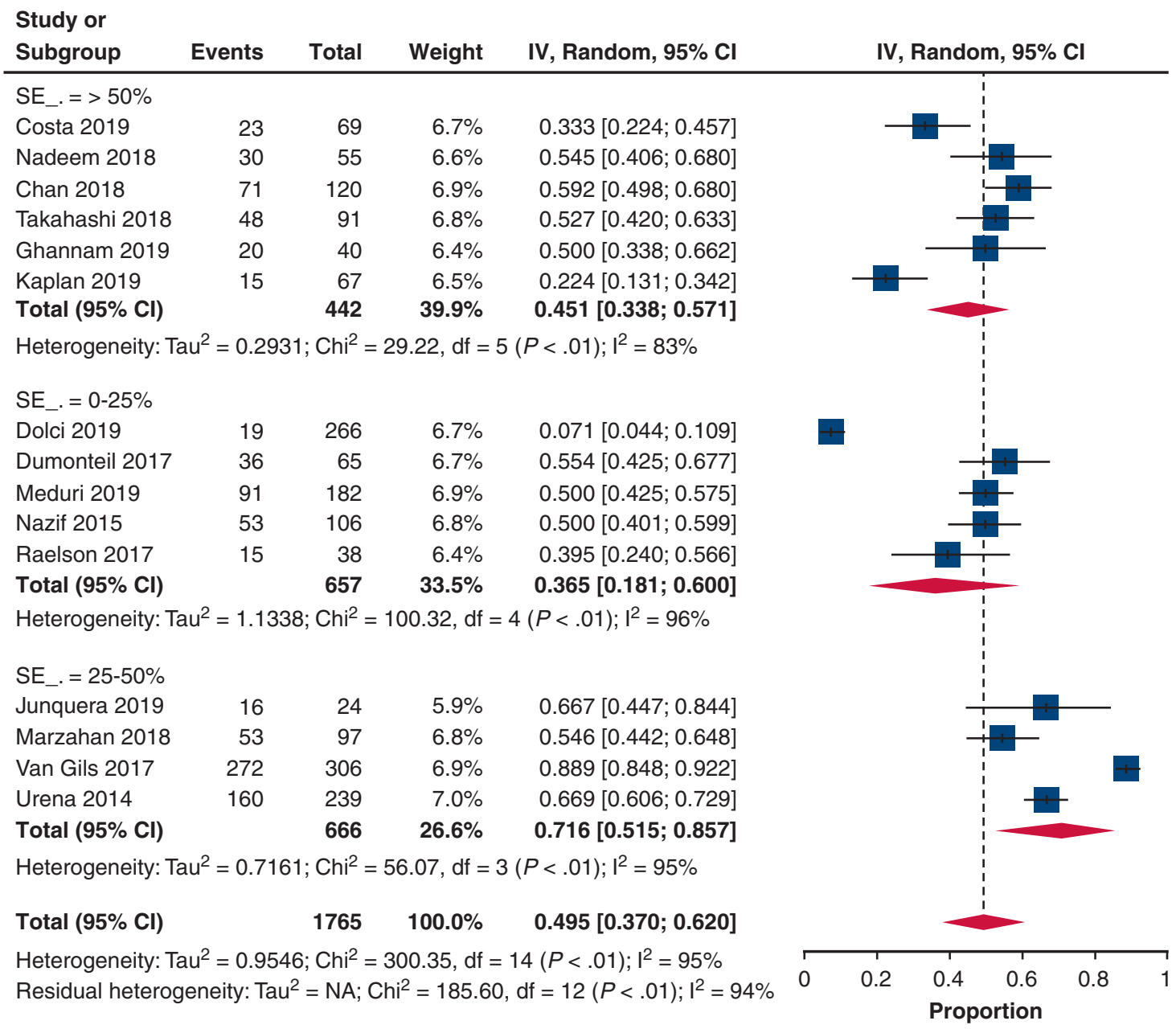

FIGURE E4. Forest plot pooling pacemaker dependency according to percentage of SE prosthesis included in the study. IV, Weighted mean difference; $C I$, confidence interval; $S E$, self-expandable. 
TABLE E1. Newcastle-Ottawa Scale for the assessment of the risk of bias in individual nonrandomized studies

\begin{tabular}{|c|c|c|c|c|}
\hline Author & Score & Selection & Comparability & Outcome/exposure \\
\hline Bjerre Thygesen et al ${ }^{9}$ & 8 & $* * * *$ & $* *$ & $* *$ \\
\hline Urena et $\mathrm{al}^{10}$ & 9 & $* * * *$ & $* *$ & $* * *$ \\
\hline Nazif et al ${ }^{11}$ & 9 & $* * * *$ & $* *$ & $* * *$ \\
\hline Van Gils et al ${ }^{12}$ & 9 & $* * * *$ & $* *$ & $* * *$ \\
\hline Raelson et $\mathrm{al}^{13}$ & 8 & $* * * *$ & $* *$ & $* *$ \\
\hline Dumonteil et $\mathrm{al}^{14}$ & 9 & $* * * *$ & $* *$ & $* * *$ \\
\hline Kaplan et al $^{15}$ & 9 & $* * * *$ & $* *$ & $* * *$ \\
\hline Chamandi et al ${ }^{16}$ & 8 & $* * * *$ & $* *$ & $* *$ \\
\hline Gaede et $\mathrm{al}^{17}$ & 8 & $* * * *$ & $* *$ & $* *$ \\
\hline Gonska et $\mathrm{al}^{18}$ & 7 & $* * * *$ & $*$ & $* *$ \\
\hline Marzahn et al ${ }^{19}$ & 9 & $* * * *$ & $* *$ & $* * *$ \\
\hline Nadeem et $\mathrm{al}^{20}$ & 9 & $* * * *$ & $* *$ & $* * *$ \\
\hline Campelo-Parada et $\mathrm{al}^{21}$ & 8 & $* * * *$ & $* *$ & $* *$ \\
\hline Mirolo et $\mathrm{al}^{22}$ & 8 & $* * * *$ & $* *$ & $* *$ \\
\hline Van Gils et $\mathrm{al}^{23}$ & 8 & $* * * *$ & $* *$ & $* *$ \\
\hline 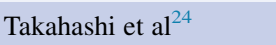 & 8 & $* * * *$ & $* *$ & $* *$ \\
\hline Chan et $\mathrm{al}^{25}$ & 9 & $* * * *$ & $* *$ & $* * *$ \\
\hline Ghannam et $\mathrm{al}^{26}$ & 9 & $* * * *$ & $* *$ & $* * *$ \\
\hline Costa et $\mathrm{al}^{27}$ & 9 & $* * * *$ & $* *$ & $* * *$ \\
\hline Dolci et $\mathrm{al}^{28}$ & 9 & $* * * *$ & $* *$ & $* * *$ \\
\hline Tovia-Brodie et $\mathrm{al}^{29}$ & 9 & $* * * *$ & $* *$ & $* *$ \\
\hline Junquera et $\mathrm{al}^{30}$ & 9 & $* * * *$ & $* *$ & $* * *$ \\
\hline Meduri et $\mathrm{al}^{31}$ & 9 & $* * * *$ & $* *$ & $* * *$ \\
\hline
\end{tabular}




\begin{tabular}{|c|c|c|c|c|c|c|c|c|c|c|c|c|c|}
\hline Study & Year & $\begin{array}{l}\text { Study design } \\
\text { (no. centers) }\end{array}$ & $\begin{array}{l}\text { Sample } \\
\text { size }\end{array}$ & $\begin{array}{l}\text { Age, } \\
\text { y }\end{array}$ & $\begin{array}{c}\text { STS } \\
\text { score, } \%\end{array}$ & $\begin{array}{c}\text { Inclusion } \\
\text { period }\end{array}$ & $\begin{array}{c}\text { Left } \\
\text { ventricle } \\
\text { ejection } \\
\text { fraction, } \\
\%\end{array}$ & $\begin{array}{c}\text { Periphera } \\
\text { artery } \\
\text { disease, } \\
\%\end{array}$ & $\begin{array}{c}\text { Diabetes } \\
\text { mellitus, } \\
\%\end{array}$ & $\begin{array}{l}\text { Valve } \\
\text { type }\end{array}$ & $\begin{array}{c}\text { Follow-up, } \\
\text { mo* }\end{array}$ & $\begin{array}{l}\text { Approach } \\
\text { for TAVI }\end{array}$ & $\begin{array}{c}\text { Mortality } \\
\text { at } 30 \mathrm{~d}\end{array}$ \\
\hline Sinhal et al ${ }^{\mathrm{E} 1}$ & 2008 & Prospective (1) & 106 & 84.2 & na & na & 62.5 & na & na & $100 \%$ SAPIEN & 1 & na & na \\
\hline Jilaihawi et al $^{\mathrm{E} 2}$ & 2009 & Retrospective (1) & 30 & 84.4 & 8.3 & $\begin{array}{l}\text { January } 2007 \\
\text { to March } 2008\end{array}$ & 47.5 & na & 17.6 & $100 \% \mathrm{MCV}$ & na & na & $8.8 \%$ \\
\hline Baan et al ${ }^{\mathrm{E} 3}$ & 2010 & Retrospective (1) & 34 & 80.4 & 5 & na & na & na & 32 & $100 \% \mathrm{MCV}$ & 1 & na & $20.5 \%$ \\
\hline Fraccaro et $\mathrm{al}^{\mathrm{E} 4}$ & 2011 & Retrospective (1) & 64 & 81 & na & $\begin{array}{l}\text { May } 2007 \\
\text { to April } 2009\end{array}$ & 52.3 & 34.4 & na & $100 \% \mathrm{MCV}$ & 12 & $\begin{array}{l}94 \% \text { transfemoral } \\
6 \% \text { transsubclavian }\end{array}$ & $5 \%$ \\
\hline Van der boon et al ${ }^{\mathrm{E} 5}$ & 2013 & Prospective (1) & 167 & 81 & na & $\begin{array}{l}\text { November } 2005 \\
\quad \text { to February } 2011\end{array}$ & 51 & 10.2 & 21.6 & $100 \% \mathrm{MCV}$ & 11,5 & $\begin{array}{l}97 \% \text { transfemoral } \\
3 \% \text { transsubclavian }\end{array}$ & na \\
\hline Pereira et $\mathrm{a}^{\mathrm{E} 6}$ & 2013 & Retrospective (1) & 65 & 79.3 & na & $\begin{array}{c}\text { August } 2007 \text { to } \\
\text { May } 2011\end{array}$ & na & 47.7 & 38.5 & $100 \% \mathrm{MCV}$ & 6 & $\begin{array}{l}78.5 \% \text { transfemoral } \\
20 \% \text { transsubclavian } \\
1.5 \% \text { direct aortic }\end{array}$ & na \\
\hline Goldenberg et $\mathrm{al}^{\mathrm{E7}}$ & 2013 & Retrospective (1) & 191 & na & na & $\begin{array}{r}\text { February } 2009 \\
\text { to July } 2012\end{array}$ & na & na & na & $\begin{array}{l}65 \% \mathrm{MCV} \\
35 \% \text { SAPIEN }\end{array}$ & 17 & na & na \\
\hline Ramazzina et a ${ }^{\mathrm{E} 8}$ & 2014 & Retrospective (1) & 97 & 83 & na & $\begin{array}{l}\text { October } 2010 \\
\text { to January } 2013\end{array}$ & 55 & na & 26.4 & $\begin{array}{l}61 \% \mathrm{MCV} \\
39 \% \text { SAPIEN }\end{array}$ & 12 & $100 \%$ transfemoral & na \\
\hline Boerlage-Van Dijk et al ${ }^{\mathrm{E} 9}$ & 2014 & Retrospective (1) & 121 & 80.5 & 4.5 & $\begin{array}{l}\text { October } 2007 \\
\text { to June } 2011\end{array}$ & na & na & 28 & $100 \% \mathrm{MCV}$ & 12 & $100 \%$ Transfemoral & na \\
\hline Renilla et a ${ }^{\mathrm{E} 10}$ & 2015 & Retrospective (1) & 95 & na & na & $\begin{array}{l}\text { January } 2007 \\
\quad \text { to December } 2011\end{array}$ & na & na & na & $100 \% \mathrm{MCV}$ & 35 & $\begin{array}{l}86.9 \% \text { transfemoral } \\
13 \% \text { transsubclavian }\end{array}$ & na \\
\hline Petronio et $\mathrm{al}^{\mathrm{E} 11}$ & 2015 & Prospective (9) & 194 & 80.2 & 7.2 & $\begin{array}{l}\text { October } 2011 \text { to } \\
\text { April } 2013\end{array}$ & na & 27.6 & 31 & $100 \% \mathrm{MCV}$ & 1 & $\begin{array}{l}89.7 \% \text { transfemoral } \\
6.2 \% \text { transsubclavian } \\
4.1 \% \text { direct aortic }\end{array}$ & $1.6 \%$ \\
\hline Weber et $\mathrm{al}^{\mathrm{E} 12}$ & 2015 & Retrospective (1) & 212 & 80.8 & 9.4 & $2008-2012$ & 52.8 & na & 25 & $100 \% \mathrm{MCV}$ & 9 & $100 \%$ transfemoral & $6.1 \%$ \\
\hline Schernthaner et $\mathrm{al}^{\mathrm{E} 13}$ & 2016 & Retrospective (1) & 153 & 81 & 6 & na & na & na & 31 & $\begin{array}{l}82 \% \mathrm{MCV} \\
18 \% \text { SAPIEN }\end{array}$ & 1,5 & $\begin{array}{l}94 \% \text { transfemoral } \\
6 \% \text { direct aortic }\end{array}$ & na \\
\hline Kostopoulou et $\mathrm{al}^{\mathrm{E} 14}$ & 2016 & Prospective (1) & 45 & 81 & na & $\begin{array}{l}\text { January } 2010 \\
\text { to February } 2012\end{array}$ & 49 & na & 27 & $100 \% \mathrm{MCV}$ & 24 & na & na \\
\hline Sideris et al ${ }^{\mathrm{E} 15}$ & 2016 & Prospective (1) & 168 & na & na & $\begin{array}{r}\text { January } 2009 \text { to } \\
\text { October } 2015\end{array}$ & na & na & na & $100 \% \mathrm{MCV}$ & na & $100 \%$ transfemoral & na \\
\hline Luke et $\mathrm{al}^{\mathrm{E} 16}$ & 2016 & Retrospective (1) & 140 & 81 & na & $\begin{array}{r}\text { July } 2011 \text { to } \\
\text { May } 2016\end{array}$ & na & na & na & $\begin{array}{l}81 \% \text { MCV } \\
19 \% \text { EVOLUT }\end{array}$ & na & $100 \%$ transfemoral & na \\
\hline Makki et al ${ }^{\mathrm{E} 17}$ & 2017 & Retrospective (1) & 172 & 83 & na & $\begin{array}{l}\text { November } 2011 \\
\text { to January } 2016\end{array}$ & 51 & na & 46 & $\begin{array}{l}92 \% \mathrm{MCV} \\
8 \% \text { LOTUS }\end{array}$ & 22 & $100 \%$ transfemoral & na \\
\hline
\end{tabular}




\begin{tabular}{|c|c|c|c|c|c|c|c|c|c|c|c|c|c|}
\hline Study & Year & $\begin{array}{l}\text { Study design } \\
\text { (no. centers) }\end{array}$ & $\begin{array}{l}\text { Sample } \\
\text { size }\end{array}$ & $\begin{array}{c}\text { Age, } \\
\mathbf{y}\end{array}$ & $\begin{array}{c}\text { STS } \\
\text { score, \% }\end{array}$ & $\begin{array}{l}\text { Inclusion } \\
\text { period }\end{array}$ & $\begin{array}{c}\text { Left } \\
\text { ventricle } \\
\text { ejection } \\
\text { fraction, } \\
\%\end{array}$ & $\begin{array}{l}\text { Peripheral } \\
\text { artery } \\
\text { disease, } \\
\%\end{array}$ & $\begin{array}{c}\text { Diabetes } \\
\text { mellitus, } \\
\%\end{array}$ & $\begin{array}{l}\text { Valve } \\
\text { type }\end{array}$ & $\begin{array}{c}\text { Follow-up, } \\
\text { mo* }\end{array}$ & $\begin{array}{l}\text { Approach } \\
\text { for TAVI }\end{array}$ & $\begin{array}{l}\text { Mortality } \\
\text { at } 30 \mathrm{~d}\end{array}$ \\
\hline Nijenhuis et $\mathrm{al}^{\mathrm{E} 18}$ & 2017 & Retrospective (1) & 155 & 80.5 & 6 & June 2007 to June 2015 & 59 & 30.3 & 34.8 & na & 24 & na & na \\
\hline Naveh et al ${ }^{\mathrm{E} 19}$ & 2017 & Prospective (1) & 110 & 80.7 & na & $\begin{array}{c}\text { September } 2008 \text { to } \\
\text { November } 2013\end{array}$ & 57.6 & 23.6 & 30 & $\begin{array}{l}75.5 \% \mathrm{MCV} \\
24.5 \% \text { SAPIEN }\end{array}$ & 12 & $\begin{array}{l}88.2 \% \text { transfemoral } \\
6.4 \% \text { transsubclavian } \\
5.5 \% \text { direct aortic }\end{array}$ & na \\
\hline Alasti et $\mathrm{al}^{\mathrm{E} 20}$ & 2018 & Prospective (1) & 152 & 83.6 & na & $\begin{array}{l}\text { April } 2012 \text { to } \\
\text { October } 2016\end{array}$ & 59.2 & 5.3 & 18.7 & $100 \%$ LOTUS & 12 & $\begin{array}{l}99.4 \% \text { transfemoral } \\
0.6 \% \text { direct aortic }\end{array}$ & $2.6 \%$ \\
\hline Ortak et $\mathrm{al}^{\mathrm{E} 21}$ & 2018 & Prospective (1) & 66 & 80.4 & 3.7 & $2014-2016$ & 53.2 & na & na & $100 \%$ LOTUS & 7 & na & $3.5 \%$ \\
\hline Rodes-Cabau et al ${ }^{\mathrm{E} 22}$ & 2018 & $\begin{array}{l}\text { Prospective } \\
\text { (11) }\end{array}$ & 103 & 80 & 5 & $\begin{array}{c}\text { June } 2014 \text { to } \\
\text { July } 2016\end{array}$ & 56 & na & 43 & $\begin{array}{l}14.5 \% \mathrm{MCV} \\
37 \% \text { EVOLUT R } \\
51.5 \% \text { SAPIEN }\end{array}$ & 12 & $\begin{array}{l}86 \% \text { transfemoral } \\
10 \% \text { transapical } \\
4 \% \text { transsubclavian }\end{array}$ & na \\
\hline Leong et $\mathrm{al}^{\mathrm{E} 23}$ & 2018 & Retrospective (1) & 67 & 80.5 & na & $\begin{array}{l}\text { January } 2013 \\
\quad \text { to December } 2015\end{array}$ & na & na & 30 & $\begin{array}{l}16.4 \% \text { EVOLUT R } \\
35.8 \% \text { MCV } \\
34.3 \% \text { SAPIEN } \\
13.4 \% \text { Others }\end{array}$ & 2,4 & na & na \\
\hline Sharma et $\mathrm{al}^{\mathrm{E} 24}$ & 2018 & Prospective (1) & 226 & 81.2 & na & $\begin{array}{l}\text { March } 2012 \text { to } \\
\text { October } 2016\end{array}$ & na & na & na & $100 \% \mathrm{BE}$ & 1 & na & na \\
\hline Bacik et $\mathrm{al}^{\mathrm{E} 25}$ & 2018 & Prospective (1) & 116 & 77.1 & na & $\begin{array}{l}\text { August } 2013 \text { to } \\
\text { Mar } 2017\end{array}$ & 50.5 & na & 40.5 & $100 \%$ SAPIEN & 12 & $\begin{array}{l}82.8 \% \text { transfemoral } \\
17.2 \% \text { transapical }\end{array}$ & na \\
\hline Megaly et $\mathrm{al}^{\mathrm{E} 26}$ & 2019 & Prospective (1) & 172 & na & na & $\begin{array}{l}\text { January } 2010 \\
\text { to May } 2017\end{array}$ & na & na & 50 & na & 12 & na & na \\
\hline Yazdchi et al ${ }^{\mathrm{E} 27}$ & 2019 & Retrospective (1) n & & na & na & $2013-2017$ & na & na & na & na & 14 & na & na \\
\hline McCaffrey et $\mathrm{al}^{\mathrm{E} 28}$ & 2019 & Retrospective (1) & 98 & 79.6 & 5 & $\begin{array}{l}\text { May } 2015 \text { to } \\
\text { March } 2018\end{array}$ & 55.7 & 26 & 35 & $100 \%$ SAPIEN & 1 & $\begin{array}{l}93 \% \text { transfemoral } \\
6 \% \text { transapical } \\
1 \% \text { direct aortic }\end{array}$ & $4.8 \%$ \\
\hline Miura et $\mathrm{al}^{\mathrm{E} 29}$ & 2019 & Retrospective (1) & 201 & 84.8 & 6.4 & $\begin{array}{l}\text { October } 2013 \\
\text { to September } \\
2016\end{array}$ & 60.6 & 9 & 26 & $100 \%$ SAPIEN & 13.5 & $\begin{array}{l}68 \% \text { transfemoral } \\
127 \% \text { transapical } \\
5 \% \text { transiliac }\end{array}$ & $0.5 \%$ \\
\hline Dhakal et $\mathrm{al}^{\mathrm{E} 30}$ & 2020 & Retrospective (1) & 176 & 80 & 5.7 & $\begin{array}{l}\text { Seprember } 2012 \\
\text { to March } 2017\end{array}$ & 53 & 31 & 43 & na & 18.9 & na & na \\
\hline Total & na & 49 centers & 3612 & 81.2 & 6 & $\begin{array}{l}\text { November } 2005 \\
\text { to March } 2018\end{array}$ & 54.4 & 24.5 & 32.1 & $\begin{array}{l}65.6 \% \text { MCV } \\
23.1 \% \text { SAPIEN } \\
8 \% \text { LOTUS } \\
2.7 \% \text { EVOLUT } \\
0.5 \% \text { Others }\end{array}$ & 11.4 & $\begin{array}{l}92.5 \% \text { transfemoral } \\
3 \% \text { transsubclavian } \\
1 \% \text { direct aortic } \\
3.2 \% \text { transapical } \\
0.3 \% \text { transiliac }\end{array}$ & $6 \%$ \\
\hline
\end{tabular}

Values are n (\%). STS score, Society of Thoracic Surgeons Risk Score; TAVI, transaortic valve implantation; na, not available; MCV, Medtronic CoreValve. *Follow-up is reported as mean or median as given by the authors. 
TABLE E3. Pacemaker details in studies including $<250$ patients $(n=30)$

\begin{tabular}{|c|c|c|c|c|c|c|c|c|c|}
\hline Study & $\begin{array}{c}\text { Indications } \\
\text { PPI }\end{array}$ & $\begin{array}{c}\text { Timing } \\
\text { of PPI, } \\
\text { d }\end{array}$ & $\begin{array}{l}\text { PPI } \\
\text { rate }\end{array}$ & $\begin{array}{l}\text { Dependency } \\
\text { definition }\end{array}$ & $\begin{array}{l}\text { Dependency } \\
\text { rate }\end{array}$ & $\begin{array}{c}\text { Dependency } \\
\text { follow-up, } \\
\text { months* }\end{array}$ & $\begin{array}{l}\text { Multivariable } \\
\text { Predictors } \\
\text { PPI }\end{array}$ & Association & $\begin{array}{c}\text { PPI-related } \\
\text { complications }\end{array}$ \\
\hline Sinhal et al ${ }^{\mathrm{E} 1}$ & na & 2 & $5.6 \%$ & na & $86 \%$ & 6 & na & na & na \\
\hline Jilaihawi et $\mathrm{al}^{\mathrm{E} 2}$ & $\begin{array}{l}90 \% \text { AVB } \\
10 \% \text { SSS }\end{array}$ & na & $33.3 \%$ & $\begin{array}{l}\text { Description by } \\
\text { case of the } \\
\% \text { ventricular } \\
\text { pacing at } \\
\text { follow-up }\end{array}$ & $66.6 \%$ & 1 & $\begin{array}{l}\text { - LBBB with left } \\
\text { axis deviation } \\
\text { - Diastolic } \\
\text { interventricular } \\
\text { septa dimension } \\
>17 \mathrm{~mm} \\
\text { - Noncoronary } \\
\text { cuspid } \\
\text { thickness } \\
>8 \mathrm{~mm}\end{array}$ & na & na \\
\hline Baan et $\mathrm{al}^{\mathrm{E} 3}$ & $100 \%$ AVB & 3 & $26.9 \%$ & $\begin{array}{l}\text { "ventricular } \\
\text { pacing" } \\
\text { reported }\end{array}$ & $100 \%$ & 1 & na & $\begin{array}{l}\text { - Smaller left } \\
\text { ventricle outflow } \\
\text { tract diameter } \\
\text { - More left-sided } \\
\text { heart axis } \\
\text { - More mitral } \\
\text { annular } \\
\text { calcification } \\
\text { - Smaller } \\
\text { postimplantation } \\
\text { indexed effective } \\
\text { orifice area }\end{array}$ & na \\
\hline Fraccaro et $\mathrm{al}^{\mathrm{E} 4}$ & $\begin{array}{l}96 \% \text { AVB } \\
4 \% \text { SSS }\end{array}$ & na & $39 \%$ & $\begin{array}{l}\text { With pacemaker } \\
\text { to VVI at the } \\
\text { lowest rate } \\
\text { possible: } \\
\text { continuous } \\
\text { pacing or } \\
\text { complete } \\
\text { AVB or AF } \\
\text { with inadequate } \\
\text { ventricular } \\
\text { response }\end{array}$ & $23.5 \%$ & 12 & $\begin{array}{l}\text { Depth of the } \\
\text { prosthesis } \\
\text { implantation } \\
\text { - Pre-existing } \\
\text { RBBB }\end{array}$ & na & na \\
\hline
\end{tabular}




\begin{tabular}{|c|c|c|c|c|c|c|c|c|c|}
\hline Study & $\begin{array}{c}\text { Indications } \\
\text { PPI }\end{array}$ & $\begin{array}{l}\text { Timing } \\
\text { of PPI, } \\
\quad \text { d }\end{array}$ & $\begin{array}{l}\text { PPI } \\
\text { rate }\end{array}$ & $\begin{array}{l}\text { Dependency } \\
\text { definition }\end{array}$ & $\begin{array}{l}\text { Dependency } \\
\text { rate }\end{array}$ & $\begin{array}{l}\text { Dependency } \\
\text { follow-up, } \\
\text { months* }\end{array}$ & $\begin{array}{c}\text { Multivariable } \\
\text { Predictors } \\
\text { PPI }\end{array}$ & Association & $\begin{array}{c}\text { PPI-related } \\
\text { complications }\end{array}$ \\
\hline Van der boon et $\mathrm{al}^{\mathrm{E} 5}$ & $\begin{array}{l}83.3 \% \text { AVB } \\
13.8 \% \text { bradycardia } \\
2.7 \% \text { others }\end{array}$ & 8 & $21.6 \%$ & $\begin{array}{l}\text { By temporarily } \\
\text { turning off } \\
\text { the PPM or } \\
\text { programming } \\
\text { to a VVI } \\
\text { modus at } \\
30 \mathrm{bpm} \text { to } \\
\text { assess } \\
\text { dependency } \\
\rightarrow \text { if high- } \\
\text { degree AVB } \\
\text { (second degree } \\
\text { Mobitz II or } \\
\text { third degree) } \\
\text { or a slow } \\
\text { (<30 bpm) } \\
\text { or absent } \\
\text { ventricular } \\
\text { escape rhythm } \\
\text { observed }\end{array}$ & $44.4 \%$ & 11.5 & na & na & na \\
\hline Pereira et $\mathrm{al}^{\mathrm{E} 6}$ & $100 \%$ AVB & 3 & $33 \%$ & $\begin{array}{l}\text { Absence of any } \\
\text { intrinsic or } \\
\text { escape rhythm } \\
\text { during back-up } \\
\text { pacing at } 30 \\
\text { beats/min (VVI) }\end{array}$ & $27 \%$ & 12 & na & $\begin{array}{l}\text { Porcelain } \\
\text { aorta }= \\
\text { independent } \\
\text { predictors of } \\
\text { pacing } \\
\text { dependency at } \\
\text { follow-up }\end{array}$ & na \\
\hline Goldenberg et al ${ }^{\mathrm{E} 7}$ & $\begin{array}{l}61.5 \% \text { AVB } \\
3 \% \text { SSS } \\
34 \% \text { others }\end{array}$ & na & $16.8 \%$ & $\begin{array}{l}\text { High degree of } \\
\text { AVB (second } \\
\text { degree or } \\
\text { complete) } \\
\text { or intrinsic } \\
\text { rhythm }<30 \\
\text { beats/min } \\
\text { during } \\
\text { pacemaker } \\
\text { inhibition }\end{array}$ & $29 \%$ & 12 & na & na & na \\
\hline
\end{tabular}


TABLE E3. Continued

\begin{tabular}{|c|c|c|c|c|c|c|c|c|c|}
\hline Study & $\begin{array}{c}\text { Indications } \\
\text { PPI }\end{array}$ & $\begin{array}{l}\text { Timing } \\
\text { of PPI, } \\
\quad \text { d }\end{array}$ & $\begin{array}{l}\text { PPI } \\
\text { rate }\end{array}$ & $\begin{array}{l}\text { Dependency } \\
\text { definition }\end{array}$ & $\begin{array}{l}\text { Dependency } \\
\text { rate }\end{array}$ & $\begin{array}{l}\text { Dependency } \\
\text { follow-up, } \\
\text { months* }\end{array}$ & $\begin{array}{c}\text { Multivariable } \\
\text { Predictors } \\
\text { PPI }\end{array}$ & Association & $\begin{array}{c}\text { PPI-related } \\
\text { complications }\end{array}$ \\
\hline Ramazzina et al $^{\mathrm{E} 8}$ & $\begin{array}{l}46 \% \text { AVB } \\
55 \% \text { others }\end{array}$ & na & $36.1 \%$ & $\begin{array}{l}>99 \% \\
\text { ventricular } \\
\text { pacing }\end{array}$ & $29 \%$ & 12 & - Use of MCV & $\begin{array}{l}\text { - Diabetes } \\
\text { - Atrial } \\
\text { fibrillation } \\
\text { before TAVI } \\
\text { - Associated } \\
\text { with no need } \\
\text { for PPI }\end{array}$ & na \\
\hline Boerlage-Van Dijk et al ${ }^{\mathrm{E} 9}$ & $91.3 \%$ AVB & 3 & $19 \%$ & $\begin{array}{l}\text { Ventricular- } \\
\text { paced rhythm } \\
\text { (no other } \\
\text { definition) }\end{array}$ & $52 \%$ & 11.3 & $\begin{array}{l}\text { - Mitral annular } \\
\text { calcification } \\
\text { - Pre-existing } \\
\text { RBBB }\end{array}$ & $\begin{array}{l}\text { - No factors } \\
\text { found }\end{array}$ & $\begin{array}{l}4.3 \% \text { atrial lead } \\
\text { repositioning } \\
4.3 \% \text { pocket } \\
\text { hematoma } \\
4.3 \% \text { cerebral } \\
\text { vascular } \\
\text { accident }\end{array}$ \\
\hline Renilla et al ${ }^{\mathrm{E} 10}$ & $100 \%$ AVB & 2 & $37.9 \%$ & $\begin{array}{l}\text { Presence of a } \\
\text { high-degree } \\
\text { AVB (Mobitz } \\
\text { II and III) or } \\
\text { a slow <30 } \\
\text { bpm or absent } \\
\text { ventricular } \\
\text { escape rhythm } \\
\text { (pacemaker } \\
\text { turned off or } \\
\text { programmed } \\
\text { to VVI modus } \\
\text { at } 30 \text { bpm) }\end{array}$ & $39.1 \%$ & 35 & na & na & $\begin{array}{l}3 \% \text { pacemaker } \\
\text { pocket } \\
\text { infection }\end{array}$ \\
\hline Petronio et al $^{\mathrm{E} 11}$ & na & na & $24.4 \%$ & $\begin{array}{l}\text { VVI } \\
\text { programming } \\
30 \text { beats/min }\end{array}$ & $40.7 \%$ & 1 & $\begin{array}{l}\text { - Implantation } \\
\text { depth }\end{array}$ & $\begin{array}{l}\text { - Implantation } \\
\text { depth <4 mm }\end{array}$ & na \\
\hline Weber et $\mathrm{al}^{\mathrm{E} 12}$ & $\begin{array}{l}90 \% \text { AVB } \\
2 \% \text { bradycardy } \\
2 \% \text { SSS } \\
5 \% \text { others }\end{array}$ & na & $23 \%$ & $\begin{array}{l}\text { Pacemaker is } \\
\text { partially or } \\
\text { frequently } \\
\text { needed to } \\
\text { ensure } \\
\text { heartbeat }\end{array}$ & $35 \%$ & 9 & na & na & na \\
\hline
\end{tabular}




\begin{tabular}{|c|c|c|c|c|c|c|c|c|c|}
\hline Study & $\begin{array}{c}\text { Indications } \\
\text { PPI }\end{array}$ & $\begin{array}{c}\text { Timing } \\
\text { of PPI, } \\
\text { d } \\
\end{array}$ & $\begin{array}{l}\text { PPI } \\
\text { rate }\end{array}$ & $\begin{array}{c}\text { Dependency } \\
\text { definition }\end{array}$ & $\begin{array}{c}\text { Dependency } \\
\text { rate }\end{array}$ & $\begin{array}{c}\text { Dependency } \\
\text { follow-up, } \\
\text { months* }\end{array}$ & $\begin{array}{c}\text { Multivariable } \\
\text { Predictors } \\
\text { PPI } \\
\end{array}$ & Association & $\begin{array}{c}\text { PPI-related } \\
\text { complications }\end{array}$ \\
\hline Schernthaner et al ${ }^{\mathrm{E} 13}$ & $\begin{array}{l}78 \% \text { AVB } \\
7 \% \text { SSS } \\
13 \% \text { Others }\end{array}$ & 7 & $20 \%$ & $\begin{array}{l}\text { Absence of } \\
\text { an escape or intrinsic } \\
\text { rhythm for } \\
30 \mathrm{~s} \text { during } \\
\text { temporary } \\
\text { back-up } \\
\text { pacing at a } \\
\text { rate of } 30 \mathrm{bpm}\end{array}$ & $37 \%$ & 1.5 & na & na & na \\
\hline Kostopoulou et al ${ }^{\mathrm{E} 14}$ & $100 \%$ AVB & na & $22 \%$ & $\begin{array}{l}\text { Asystole or } \\
\text { CHB with } \\
\text { or without } \\
\text { escape } \\
\text { rhythm after } \\
\text { cessation } \\
\text { of pacing }\end{array}$ & $9 \%$ & 12 & $\begin{array}{l}\text { - Prolonged } \\
\text { HV interval } \\
\text { prognostic } \\
\text { for PPI }\end{array}$ & $\begin{array}{l}\text { - Trend between } \\
\Delta \text { QRS and PPI } \\
\text { at 6-mo analysis }\end{array}$ & na \\
\hline Sideris et $\mathrm{al}^{\mathrm{E} 15}$ & $100 \%$ AVB & na & $38.7 \%$ & $\begin{array}{l}\text { High } \\
\text { ventricular } \\
\text { pacing rate } \\
>99 \%\end{array}$ & $100 \%$ & 6 & na & na & $\begin{array}{l}1.5 \% \\
\text { pneumothorax }\end{array}$ \\
\hline Luke et al ${ }^{\mathrm{E} 16}$ & $100 \%$ AVB & na & $39.3 \%$ & "Pacing" & $0.7 \%$ & 3 & $\begin{array}{l}\text { - Previous } \\
\text { conduction } \\
\text { system disease } \\
\text { - History of } \\
\text { atrial } \\
\text { fibrillation }\end{array}$ & $\begin{array}{l}\text { Trends with } \\
\text { - history of atrial } \\
\text { fibrillation } \\
\text { - presence of } \\
\text { RBBB } \\
\text { - male sex } \\
\text { - atrioventricular } \\
\text { nodal blocking } \\
\text { drugs }\end{array}$ & na \\
\hline Makki et al ${ }^{\mathrm{E} 17}$ & $\begin{array}{l}63 \% \text { AVB } \\
4 \% \text { bradycardia } \\
33 \% \text { others }\end{array}$ & 3 & $14 \%$ & $\begin{array}{l}\text { Underlying } \\
\text { ventricular } \\
\text { asystole }>5 \mathrm{~s}, \\
\text { CHB, }>50 \% \\
\text { pacing, } \\
\text { symptoms in } \\
\text { the setting of } \\
\text { bradycardia } \\
\text { (rate }<50 \mathrm{bpm} \text { ) }\end{array}$ & $33 \%$ & 3 & na & na & na \\
\hline
\end{tabular}


TABLE E3. Continued

\begin{tabular}{|c|c|c|c|c|c|c|c|c|c|}
\hline Study & $\begin{array}{c}\text { Indications } \\
\text { PPI }\end{array}$ & $\begin{array}{l}\text { Timing } \\
\text { of PPI, } \\
\quad \text { d }\end{array}$ & $\begin{array}{l}\text { PPI } \\
\text { rate }\end{array}$ & $\begin{array}{l}\text { Dependency } \\
\text { definition }\end{array}$ & $\begin{array}{l}\text { Dependency } \\
\text { rate }\end{array}$ & $\begin{array}{c}\text { Dependency } \\
\text { follow-up, } \\
\text { months* }\end{array}$ & $\begin{array}{c}\text { Multivariable } \\
\text { Predictors } \\
\text { PPI }\end{array}$ & Association & $\begin{array}{c}\text { PPI-related } \\
\text { complications }\end{array}$ \\
\hline Nijenhuis et al ${ }^{\mathrm{E} 18}$ & $87 \%$ AVB & 8 & $24 \%$ & $\begin{array}{l}\text { Ventricular } \\
\text { pacing } \\
\text { reported }\end{array}$ & $68 \%$ & 27 & $\begin{array}{l}\text { - Previous atrial } \\
\text { fibrillation } \\
\text { - Use of digoxin } \\
\text { - MCV } \\
\text { implantation } \\
\text { - Left heart axis }\end{array}$ & na & na \\
\hline Naveh et al ${ }^{\mathrm{E} 19}$ & $100 \%$ AVB & na & $34.5 \%$ & $\begin{array}{l}\text { Absent or } \\
\text { inadequate } \\
\text { intrinsic } \\
\text { ventricular } \\
\text { rhythm on } \\
\text { pacemaker } \\
\text { interrogation } \\
\text { (intrinsic } \\
\text { rhythm }<30 \\
\text { bpm); }>5 \% \\
\text { VP from the } \\
\text { last follow- } \\
\text { up on } \\
\text { pacemaker } \\
\text { interrogation; } \\
\text { any evidence } \\
\text { of VP on } \\
\text { pacemaker } \\
\text { interrogation } \\
\text { in case } \\
\text { where the } \\
\text { programmed } \\
\text { AV interval } \\
\text { was }>300 \text { ms }\end{array}$ & $68.4 \%$ & 12 & $\begin{array}{l}\text { - Baseline RBBB } \\
\text { - PR interval } \\
\text { (each } \\
\text { increment of } \\
10 \text { milliseconds } \\
\text { in PR interval, } \\
\text { risk for PPI } \\
17 \% \text { greater) }\end{array}$ & na & $0 \%$ \\
\hline
\end{tabular}




\begin{tabular}{|c|c|c|c|c|c|c|c|c|c|}
\hline Study & $\begin{array}{c}\text { Indications } \\
\text { PPI }\end{array}$ & $\begin{array}{c}\text { Timing } \\
\text { of PPI, } \\
\text { d }\end{array}$ & $\begin{array}{l}\text { PPI } \\
\text { rate }\end{array}$ & $\begin{array}{c}\text { Dependency } \\
\text { definition }\end{array}$ & $\begin{array}{l}\text { Dependency } \\
\text { rate }\end{array}$ & $\begin{array}{c}\text { Dependency } \\
\text { follow-up, } \\
\text { months* }\end{array}$ & $\begin{array}{c}\text { Multivariable } \\
\text { Predictors } \\
\text { PPI }\end{array}$ & Association & $\begin{array}{l}\text { PPI-related } \\
\text { complications }\end{array}$ \\
\hline Alasti et al ${ }^{\mathrm{E} 20}$ & $\begin{array}{l}89.2 \% \text { AVB } \\
2.6 \% \text { SSS } \\
7.8 \% \text { Others }\end{array}$ & 3 & $25 \%$ & $\begin{array}{l}\text { The need for } \\
\text { ventricular } \\
\text { pacing when } \\
\text { the pacing rate } \\
\text { was lowered } \\
\text { to } 40 \mathrm{bpm} \text { for } \\
10 \mathrm{~s} \rightarrow \\
\text { dependent: } \\
\text { slow }(<40 \mathrm{bpm}) \\
\text { or absent } \\
\text { ventricular } \\
\text { escape rhythm } \\
\text { or AVB (Mobitz } \\
\text { II or III) }\end{array}$ & $38 \%$ & 12 & na & na & $\begin{array}{l}16 \% \\
\text { hematomas }\end{array}$ \\
\hline Ortak et al ${ }^{\mathrm{E} 21}$ & $\begin{array}{l}83 \% \text { AVB } \\
16 \% \text { others }\end{array}$ & na & $22 \%$ & na & $64 \%$ & 1 & $\begin{array}{l}\text { - Implantation } \\
\text { depth } \\
\text { - LOTUS } \\
\text { implantation } \\
\text { depth }>4.8 \mathrm{~mm} \\
=\text { cut-off to } \\
\text { predict PPI }\end{array}$ & na & na \\
\hline
\end{tabular}

\begin{tabular}{|c|c|c|c|c|c|c|c|c|c|}
\hline $\begin{array}{l}\text { Rodes-Cabau } \\
\text { et } \mathrm{al}^{\mathrm{E} 22}\end{array}$ & $\begin{array}{l}81 \% \text { AVB } \\
9 \% \\
\text { bradycardia } \\
9 \% \text { others }\end{array}$ & 42 & $11 \%$ & na & $78 \%$ & 12 & na & na & na \\
\hline Leong et $\mathrm{al}^{\mathrm{E} 23}$ & $\begin{array}{l}74.6 \% \text { AVB } \\
20.8 \% \\
\text { bradycardia } \\
13.5 \% \text { others }\end{array}$ & 2.3 & $44.8 \%$ & $\begin{array}{l}\text { Ventricular } \\
\text { pacing } \\
\text { reported }\end{array}$ & $73 \%$ & 2.4 & na & $\begin{array}{l}\text { - Male sex } \\
\text { - Increase in } \\
\text { QRS duration } \\
\text { post-TAVI } \\
\text { - Associated } \\
\text { with PPI }\end{array}$ & $0 \%$ \\
\hline
\end{tabular}


TABLE E3. Continued

\begin{tabular}{|c|c|c|c|c|c|c|c|c|c|}
\hline Study & $\begin{array}{c}\text { Indications } \\
\text { PPI }\end{array}$ & $\begin{array}{c}\text { Timing } \\
\text { of PPI, } \\
\text { d } \\
\end{array}$ & $\begin{array}{l}\text { PPI } \\
\text { rate }\end{array}$ & $\begin{array}{c}\text { Dependency } \\
\text { definition }\end{array}$ & $\begin{array}{c}\text { Dependency } \\
\text { rate }\end{array}$ & $\begin{array}{c}\text { Dependency } \\
\text { follow-up, } \\
\text { months* }\end{array}$ & $\begin{array}{c}\text { Multivariable } \\
\text { Predictors } \\
\text { PPI }\end{array}$ & Association & $\begin{array}{c}\text { PPI-related } \\
\text { complications }\end{array}$ \\
\hline Sharma et $\mathrm{al}^{\mathrm{E} 24}$ & na & na & $11.1 \%$ & $\begin{array}{l}\text { Spontaneous } \\
\text { response } \\
\text { ventricular } \\
\text { rate less } \\
\text { than } 30 \mathrm{bpm} \\
\text { during } \\
\text { backup } \\
\text { pacing set } \\
\text { at } 30 \text { beats/ } \\
\text { min for } 30 \mathrm{~s}\end{array}$ & $32 \%$ & 1 & na & $\begin{array}{l}\text { - RBBB } \\
\text { - Intra- } \\
\text { procedural } \\
\text { CHB } \\
\text { - Bifascicular } \\
\text { block } \\
\text { - QRS duration } \\
>120 \mathrm{~ms} \\
\text { - All associated } \\
\text { with pacing } \\
\text { dependence } \\
\text { at } 30 \mathrm{~d}\end{array}$ & na \\
\hline Bacik et al ${ }^{\mathrm{E} 25}$ & $\begin{array}{l}49.8 \% \text { AVB } \\
6.3 \% \text { SSS } \\
43.9 \% \text { others }\end{array}$ & 5.5 & $13.8 \%$ & $\begin{array}{l}\text { More than } \\
95 \% \text { pacing } \\
\text { events }\end{array}$ & $12 \%$ & 12 & $\begin{array}{l}\text { - Weight } \\
\text { - Absence } \\
\text { of AF } \\
\text { - Aortic peak } \\
\text { gradient } \\
\text { - Aortic } \\
\text { valve area } \\
\text { - Severity of } \\
\text { pulmonary } \\
\text { hypertension }\end{array}$ & na & na \\
\hline Megaly et al ${ }^{\mathrm{E} 26}$ & $\begin{array}{l}50.6 \% \text { AVB } \\
34.9 \% \text { others }\end{array}$ & na & $35 \%$ & $\begin{array}{l}\text { CHB } \\
\text { requiring } \\
\text { ventricular } \\
\text { pacing }\end{array}$ & $3.5 \%$ & 12 & na & na & na \\
\hline Yazdchi et al ${ }^{\mathrm{E} 27}$ & $78 \%$ AVB & 2 & $8.7 \%$ & $\begin{array}{l}\text { Ventricular } \\
\text { pacing } \\
\text { reported }\end{array}$ & $87 \%$ & 14 & na & na & na \\
\hline
\end{tabular}


TABLE E3. Continued

\begin{tabular}{|c|c|c|c|c|c|c|c|c|c|}
\hline Study & $\begin{array}{c}\text { Indications } \\
\text { PPI }\end{array}$ & $\begin{array}{c}\text { Timing } \\
\text { of PPI, } \\
\text { d }\end{array}$ & $\begin{array}{l}\text { PPI } \\
\text { rate }\end{array}$ & $\begin{array}{l}\text { Dependency } \\
\text { definition }\end{array}$ & $\begin{array}{l}\text { Dependency } \\
\text { rate }\end{array}$ & $\begin{array}{c}\text { Dependency } \\
\text { follow-up, } \\
\text { months* }\end{array}$ & $\begin{array}{c}\text { Multivariable } \\
\text { Predictors } \\
\text { PPI }\end{array}$ & Association & $\begin{array}{c}\text { PPI-related } \\
\text { complications }\end{array}$ \\
\hline $\begin{array}{l}\text { McCaffrey } \\
\text { et al } \mathrm{al}^{\mathrm{E} 28}\end{array}$ & na & na & $11.2 \%$ & $\begin{array}{l}\text { Ventricular } \\
\text { pacing } \\
\text { reported }\end{array}$ & $75 \%$ & 1 & na & $\begin{array}{l}\text { - Predictive } \\
\text { factors of } \\
\text { acute } \\
\text { conduction } \\
\text { abnormalities } \\
\text { (4) } \\
\text { - Predictive } \\
\text { factors of } \\
\text { new } \\
\text { conductions } \\
\text { abnormalities } \\
\text { after discharge } \\
\text { (5) }\end{array}$ & na \\
\hline Miura et al ${ }^{\mathrm{E} 29}$ & $\begin{array}{l}90 \% \text { AVB } \\
10 \% \text { SSS }\end{array}$ & 6 & $5 \%$ & $\begin{array}{l}\text { Ventricular } \\
\text { pacing } \\
\text { reported }\end{array}$ & $40 \%$ & 12 & na & na & na \\
\hline Dhakal et al ${ }^{\mathrm{E} 30}$ & $\begin{array}{l}80 \% \text { AVB } \\
17 \% \text { SSS } \\
3 \% \text { others }\end{array}$ & 2 & $17 \%$ & $\begin{array}{l}\text { Ventricular } \\
\text { pacing rate }\end{array}$ & $54 \%$ & 2.7 & RBBB & $\begin{array}{l}\text { In univariate } \\
\text { analysis: } \\
\text { - Valve size } \\
\text { - SE valve } \\
\text { - RBBB } \\
\text { - Prolonged } \\
\text { PR interval }\end{array}$ & na \\
\hline Total & $\begin{array}{l}72 \% \text { AVB } \\
1.7 \% \text { SSS } \\
3 \% \\
\text { bradycardia } \\
23.2 \% \text { others }\end{array}$ & 6,5 & $23.8 \%$ & na & na & 9 & na & na & na \\
\hline $\begin{array}{l}\text { alues are n }(\%) . P F \\
\text { lock; } P P M \text {, perman }\end{array}$ & $\begin{array}{l}\text { er implantation; } n a \text {, } \\
\text { ker; } M C V \text {, Medtron }\end{array}$ & $\begin{array}{l}\text { aailable; } A \\
\text { reValve; } 7\end{array}$ & $\begin{array}{l}B, \text { atriovent } \\
I V I \text {, transcat }\end{array}$ & $\begin{array}{l}\text { icular block; } S S S, \text { sick } \\
\text { eter aortic valve impla }\end{array}$ & $\begin{array}{l}\text { yndrome; } \angle B B B, 1 \\
\text { i; } C H B \text {, complete }\end{array}$ & $\begin{array}{l}\text { eft bundle branc } \\
\text { heart block; } S E\end{array}$ & $\begin{array}{l}\text { lock; } V V I, \text { single-chambe } \\
\text { elf-expandable. *Follow-u }\end{array}$ & $\begin{array}{l}F, \text { atrial fibrillation; } R \\
\text { ted as mean or median }\end{array}$ & $\begin{array}{l}B \text {, right bundle branch } \\
\text { given by the authors. }\end{array}$ \\
\hline
\end{tabular}


TABLE E4. Leave-one-study out analysis

\begin{tabular}{|c|c|c|c|}
\hline Left-out study & OR & $95 \%$ CI & $P$ value \\
\hline \multicolumn{4}{|l|}{ RBBB } \\
\hline Kaplan $2019^{15}$ & 2.3798 & $1.1797-4.8008$ & .0155 \\
\hline Nadeem $2018^{20}$ & 2.2425 & $1.0932-4.6002$ & .0276 \\
\hline Raelson $2017^{13}$ & 2.0960 & $1.0400-4.2241$ & .0385 \\
\hline Costa $2019^{27}$ & 1.9345 & $1.0126-3.9288$ & .0479 \\
\hline Meduri $2019^{31}$ & 1.8850 & $1.0136-3.7261$ & .0483 \\
\hline Chan $2018^{25}$ & 1.6386 & 0.8001-3.3559 & .1770 \\
\hline \multicolumn{4}{|l|}{$\mathrm{AF}$} \\
\hline Kaplan $2019^{15}$ & 1.2220 & $0.4251-3.5122$ & .7098 \\
\hline Nadeem $2018^{20}$ & 0.9829 & $0.3378-2.8599$ & .9748 \\
\hline Raelson $2017^{13}$ & 0.9094 & $0.2919-2.8336$ & .8699 \\
\hline Chan $2018^{25}$ & 1.4551 & $0.5188-4.0811$ & .4760 \\
\hline \multicolumn{4}{|l|}{ SE } \\
\hline Kaplan $2019^{15}$ & 1.9233 & $1.0326-3.7266$ & .0426 \\
\hline Nadeem $2018^{20}$ & 2.3432 & $1.1402-4.8153$ & .0205 \\
\hline Raelson $2017^{13}$ & 2.5419 & $1.3676-4.7244$ & .0032 \\
\hline Van Gils $2017^{12}$ & 2.3947 & $1.1342-5.0562$ & .0220 \\
\hline Urena $2014^{10}$ & 1.8686 & $0.8743-3.9938$ & .1067 \\
\hline Takahashi $2018^{24}$ & 1.8711 & $1.0048-3.4844$ & .0483 \\
\hline
\end{tabular}

$O R$, Odds ratio; $C I$, confidence interval; $R B B B$, right bundle branch block; $A F$, atrial fibrillation; $S E$, self-expandable. 